\title{
Supernovae and their host galaxies - VII. The diversity of Type Ia supernova progenitors
}

\author{
A. A. Hakobyan, ${ }^{1 \star}$ L. V. Barkhudaryan, ${ }^{1 \star}$ A. G. Karapetyan, ${ }^{1}$ M. H. Gevorgyan, ${ }^{1}$ \\ G. A. Mamon, ${ }^{2}$ D. Kunth, ${ }^{2}$ V. Adibekyan ${ }^{3}$ and M. Turatto ${ }^{4}$ \\ ${ }^{1}$ Center for Cosmology and Astrophysics, Alikhanian National Science Laboratory, 2 Alikhanian Brothers Str., 0036 Yerevan, Armenia \\ ${ }^{2}$ Institut d'Astrophysique de Paris (UMR 7095: CNRS \& Sorbonne Université), 98 bis bd Arago, F-75014 Paris, France \\ ${ }^{3}$ Instituto de Astrofísica e Ciência do Espaço, Universidade do Porto, CAUP, Rua das Estrelas, P-4150-762 Porto, Portugal \\ ${ }^{4}$ INAF - Osservatorio Astronomico di Padova, Vicolo dell'Osservatorio 5, I-35122 Padova, Italy
}

Accepted 2020 September 21. Received 2020 September 15; in original form 2020 July 20

\begin{abstract}
We present an analysis of the light curve (LC) decline rates $\left(\Delta m_{15}\right)$ of 407 normal and peculiar supernovae (SNe) Ia and global parameters of their host galaxies. As previously known, there is a significant correlation between the $\Delta m_{15}$ of normal SNe Ia and global ages (morphologies, colours, masses) of their hosts. On average, those normal SNe Ia that are in galaxies from the Red Sequence (early-type, massive, old hosts) have faster declining LCs in comparison with those from the Blue Cloud (latetype, less massive, younger hosts) of the colour-mass diagram. The observed correlations between the $\Delta m_{15}$ of normal SNe Ia and hosts' parameters appear to be due to the superposition of at least two distinct populations of faster and slower declining normal SNe Ia from older and younger stellar components. We show, for the first time, that the $\Delta m_{15}$ of 91bg-and 91T-like $\mathrm{SNe}$ is independent of host morphology and colour. The distribution of hosts on the colour-mass diagram confirms the known tendency for $91 \mathrm{bg}$-like SNe to occur in globally red/old galaxies while 91T-like events prefer blue/younger hosts. On average, the youngest global ages of 02cx-like SNe hosts and their positions in the colour-mass diagram hint that these events likely originate from young population, but they differ from 91T-like events in the LC decline rate. Finally, we discuss the possible explosion channels and present our favoured SN Ia models that have the potential to explain the observed SN-host relations.
\end{abstract}

Key words: supernovae: individual: Type Ia - galaxies: evolution - galaxies: star formation - galaxies: stellar content.

\section{INTRODUCTION}

In the realm of stellar explosions, Type Ia supernovae ( $\mathrm{SNe}$ Ia) are believed to be the evolutionary endpoint, accompanied by the thermonuclear explosion, of carbon-oxygen (CO) white dwarf (WD) stars in interacting close binary systems (e.g. Maeda \& Terada 2016). These events play a key role in understanding the evolution of binary stellar systems (e.g. Ruiter, Belczynski \& Fryer 2009), the chemical enrichment of galaxies (e.g. Matteucci \& Greggio 1986), and the nature of accelerating expansion of the Universe (e.g. Riess et al. 1998; Perlmutter et al. 1999).

It is now clear that Type Ia $\mathrm{SNe}$ are not a homogeneous population of WD explosions, as they display a variety of photometric and spectroscopic properties (see reviews by Taubenberger 2017; Jha 2017). In the local Universe, in comparison with moderately uniform normal SNe Ia (Branch, Fisher \& Nugent 1993), about one third of all SNe Ia events have peculiar characteristics (e.g. Li et al. 2011; Graur et al. 2017). The most common subclasses of peculiar SNe Ia are: $i$ ) 91T-like events (Filippenko et al. 1992b; Phillips et al. 1992), which are overluminous at the $B$-band maximum $(\sim 0.6 \mathrm{mag}$ more luminous than normal SNe Ia) and have slow-declining light curves (LCs), with distinctive pre-peak spectra dominated by Fe III

\footnotetext{
^ E-mail: artur.hakobyan@yerphi.am (AAH); 1.barkhudaryan@yerphi.am (LVB)
}

lines, ii) 91bg-like SNe (Filippenko et al. 1992a; Leibundgut et al. 1993; Turatto et al. 1996), which are subluminous events ( 2 mag less luminous than normal ones) and have fast-declining LCs, with post-maximum spectra dominated by unusually strong O I and Ti II lines, and iii) low luminosity (more than 2 mag fainter than normal $\mathrm{SNe} \mathrm{Ia}$ ) and faster declining 02cx-like $\mathrm{SNe}$ (also called SNe Iax, $\mathrm{Li}$ et al. 2003; Foley et al. 2013), with early spectra resembling those of 91T-like events. There is also a tiny percentage of other peculiar SNe Ia, including the faint but slowly declining 02es-like SNe, so called Ca-rich transients, the extremely luminous 06gz-like (also called super-Chandrasekhar) $\mathrm{SNe}$, and other SNe Ia with spectra showing evidence of interaction with the circumstellar medium (see e.g. Taubenberger 2017, for a review on the extremes of SNe Ia).

In general, the behaviour of the LCs of SN Ia depends on the mass of synthesized radioactive ${ }^{56} \mathrm{Ni}$, the kinetic energy of the explosion, and the opacity of the ejecta (e.g. Arnett 1982; Mazzali et al. 2007). Importantly, Type Ia $\mathrm{SNe}$ show a key relation between their luminosity at the $B$-band maximum and their LC decline rate $\Delta m_{15}$ (i.e. magnitude difference between the maximum and 15 days after, Phillips 1993). This is known as the width-luminosity relation (faster declining $\mathrm{SNe}$ Ia are fainter) that played an enormous role in standardization of SNe Ia and their use in cosmology as the best distance indicators. However, the width-luminosity relation is well established for normal SNe Ia, while peculiar events deviate, some- 
times very strongly, from that relation (see e.g. Ashall et al. 2016; Taubenberger 2017). Note that the $\Delta m_{15}$ and colours of SNe Ia are also related: the faster declining events correspond to the intrinsically redder SNe (e.g. Phillips et al. 1999).

Theoretically, there are many possibilities in the proposed progenitor channels for Type Ia SNe that are still under debate (see e.g. Livio \& Mazzali 2018; Ruiter 2020), however they are generally categorized into the following main classes; the single-degenerate (SD) and double-degenerate (DD) channels, both of which probably occur in nature (e.g. Hillebrandt et al. 2013). In the SD channel (e.g. Whelan \& Iben 1973), a degenerate WD grows in mass through accretion from a non-degenerate companion, consequently causing an explosion when the WD mass reaches the Chandrasekhar mass limit $\left(\approx 1.4 M_{\odot}\right)$. The non-degenerate companion can be a mainsequence/subgiant star, or a red giant, or a helium (He) star (e.g. Nomoto, Iwamoto \& Kishimoto 1997). In the DD channel (e.g. Iben \& Tutukov 1984, 1985), an explosion occurs when two degenerate WDs coalescence or interact through accretion with each other in a binary system, after having been brought together due to the loss of orbital angular momentum via the emission of gravitational waves.

Many studies attempted to constrain the nature of $\mathrm{SNe} \mathrm{Ia}$ progenitors by exploring the relations between the spectroscopic/photometric properties of SNe Ia and the global characteristics of their host galaxies (or of the site of explosion), such as morphology, colour, star formation rate (SFR), mass, metallicity, and age of the stellar population (e.g. Hamuy et al. 2000; Howell 2001; Gallagher et al. 2005; Howell et al. 2009; Neill et al. 2009; Sullivan et al. 2010; González-Gaitán et al. 2011; Gupta et al. 2011; Johansson et al. 2013; Pan et al. 2014; Ashall et al. 2016; Roman et al. 2018; Meng 2019; Pruzhinskaya et al. 2020). In short, these studies showed that more luminous and slower declining SNe Ia explode, on average, in galaxies with later morphological type, lower mass, higher specific SFR, and younger stellar population age (for SN local environment as well). Kang et al. (2020) recently claimed a significant correlation between the SN Ia luminosity (or LC decline rate) and the stellar population age of its host, at a 99.5 per cent confidence level. They suggested that the previously reported correlations with host morphology, mass, and SFR are originated from the difference in population age (see also Rigault et al. 2013; Childress et al. 2014; Rose et al. 2019). However, SN Ia samples in these studies consist only of spectroscopically normal events with known LC properties, or sometimes include only a tiny portion of peculiar SNe Ia. Therefore, the relations between LC properties of peculiar $\mathrm{SNe}$ Ia and the characteristics of their host galaxies have not been explored in such detail as it was done for normal SNe Ia.

In our recent paper (Barkhudaryan et al. 2019), we comparatively studied elliptical host galaxies (distances $\leqslant 150 \mathrm{Mpc}$ ) of 66 normal SNe Ia and 41 subluminous/91bg-like events from the footprint of the Sloan Digital Sky Survey (SDSS), without considering SN Ia LC properties. Our results supported the earlier suggestion (e.g. Gallagher et al. 2005, 2008) that the characteristics of normal SNe Ia and $91 \mathrm{bg}$-like events depend more on age than on mass or metallicity of the elliptical host galaxies. We showed, that the majority of the elliptical hosts of 91bg-like events are very old ( $>8 \mathrm{Gyr}$ ) in comparison with those of normal SNe Ia, which are on average bluer and might have more residual star formation that gives rise to younger/prompt SNe Ia progenitors. In other words, we showed that the age distribution of $91 \mathrm{bg}$-like $\mathrm{SNe}$ hosts does not extend down to the stellar ages that produce significant $u$-band fluxes in early-type hosts of normal SNe Ia, thus younger stars in these hosts do not pro- duce $91 \mathrm{bg}$-like SNe. Therefore, we concluded that the delay time ${ }^{1}$ distribution (DTD) of $91 \mathrm{bg}$-like SNe is likely weighted toward long delay times, larger than several Gyr (see also Crocker et al. 2017; Panther et al. 2019). These results led us to favour SN Ia progenitor models such as He-ignited violent mergers (e.g. Pakmor et al. 2013) that have the potential to explain the different DTDs of normal SNe Ia and 91bg-like events.

In the present study, we significantly improved the inclusion of various spectroscopic subclasses of Type Ia SNe (normal SN Ia and peculiar 91T-, 91bg- and 02cx-like events) from host galaxies with almost all Hubble morphological types, including objects not only from the SDSS, but also from the Panoramic Survey Telescope and Rapid Response System (Pan-STARRS) and the SkyMapper Southern Sky Survey, thus covering the entire sky. In addition, instead of relying only on the discrete spectroscopic classifications, $\mathrm{SNe}$ Ia in our sample now have the available continuous and extinctionindependent LC decline rate $\left(\Delta m_{15}\right)$ values in the $B$-band. The goals of this paper are to properly identify the diversity of SNe Ia and better constrain the nature (i.e. the progenitor channel, through the DTD) of their different subclasses through a comprehensive study of SN Ia LC decline rates and global properties of their hosts (e.g. morphology, stellar mass, colour, and age of stellar population) in a well-defined sample of more than $400 \mathrm{SNe}$ Ia from relatively nearby galaxies.

This is the seventh paper of the series, the content of which is as follows. Section 2 presents the sample selection and reduction. Our results and interpretations are presented in Section 3. Finally, Section 4 summarizes our conclusions. To conform to data values used in our series of the papers, a Hubble constant $H_{0}=$ $73 \mathrm{~km} \mathrm{~s}^{-1} \mathrm{Mpc}^{-1}$ is adopted.

\section{SAMPLE SELECTION AND REDUCTION}

\subsection{SN Ia sample}

We used the Open Supernova Catalog (Guillochon et al. 2017) to collect spectroscopically classified $\mathrm{SNe}$ Ia with distances $\leqslant 150 \mathrm{Mpc}(z \lesssim 0.036){ }^{2}$ discovered up to 2019 May 1 . All SNe Ia are required to have a $B$-band LC decline rate $\left(\Delta m_{15}\right)$. We compiled the LC decline rates with their errors from various publications where different LC fitters were applied on the $B$-band photometry (or measured directly from the LCs) with wide temporal coverage for individual SNe (e.g. Hicken et al. 2009; Ganeshalingam et al. 2010; Folatelli et al. 2013; Ashall et al. 2016; Stahl et al. 2019). Note that the $B$-band is historically the most often used in SN studies, and therefore the data is best sampled in this band. It should be noted also that the $\Delta m_{15}$ of an SN Ia is a weak function of the lineof-sight dust extinction towards the $\mathrm{SN}$ in host galaxy, which affects the LCs (Phillips et al. 1999): $\Delta m_{15}^{\text {true }}=\Delta m_{15}^{\text {obs }}+0.1 E(B-V)$, where the last term in the equation contains colour excess towards the SN. The $E(B-V)$ values are mostly distributed within 0 to $0.3 \mathrm{mag}$, with the mean value of $\sim 0.1 \mathrm{mag}$ (e.g. Phillips et al. 1999; Ashall et al. 2016). Therefore, the term in the equation is between 0 to 0.03 mag, while the mean error in $\Delta m_{15}$ estimation is

\footnotetext{
1 The delay time is the time interval between the SN Ia progenitor formation and the later thermonuclear explosion of WD.

2 The luminosity distances of SNe and/or host galaxies are calculated using the recession velocities both corrected to the centroid of the Local Group and for the Virgocentric infall (see Hakobyan et al. 2012, for more details).
} 
Table 1. Numbers and fractions of SNe Ia subclasses in our (distances $\leqslant 150 \mathrm{Mpc}$ ) and LOSS (distances $\leqslant 80 \mathrm{Mpc}$ ) volume-limited samples.

\begin{tabular}{lrcrr}
\hline \multirow{2}{*}{ SN subclass } & \multicolumn{2}{c}{ Our } & \multicolumn{2}{c}{ LOSS } \\
\hline SN & \multicolumn{1}{c}{$f r$} & $N_{\mathrm{SN}}$ & \multicolumn{1}{c}{$f r$} \\
\hline normal & 303 & $73.5_{-3.0}^{+2.8}$ & 52 & $70.3_{-7.8}^{+7.0}$ \\
$91 \mathrm{~T}$ & 42 & $10.2_{-1.9}^{+2.2}$ & 7 & $9.4_{-4.1}^{+5.9}$ \\
$91 \mathrm{bg}$ & 50 & $12.1_{-2.0}^{+2.3}$ & 11 & $14.9_{-5.2}^{+6.7}$ \\
02cx & 12 & $4.1_{-1.2}^{+1.5}$ & 2 & $5.4_{-3.0}^{+5.1}$ \\
02es & 5 & - & 0 & - \\
06gz & 2 & - & & \\
All & 414 & & 74 & \\
\hline
\end{tabular}

Notes. To be comparable with the LOSS sample, the fractions (in per cent) of SN subclasses in our sample are calculated, using the approach of Cameron (2011), without two 06gz-like SNe. Despite the different nature of 02cx- and 02es-like events (White et al. 2015), the LOSS merged these $\mathrm{SNe}$ in a single bin, and we therefore presented the corresponding merged fraction in our sample as well (however the SN numbers are presented separately).

$\sim 0.1$ mag. ${ }^{3}$ For this reason, we consider the $B$-band $\Delta m_{15}$ as a practically extinction-independent parameter. In the compilation of SNe Ia LC decline rates, we avoided using any transformation from LC stretch ${ }^{4}$ to $\Delta m_{15}$, because it is unclear how reliable such a transformation is (e.g. Ashall et al. 2016; Siebert et al. 2019).

The collected SNe Ia are also required to have available subclasses of their spectroscopy (e.g. normal, 91T-, 91bg-, and 02cx-like). Following Barkhudaryan et al. (2019), we carried out an extensive literature search to compile the information on the subclasses. As an interactive archive of SN spectra and corresponding references, we mostly used the Weizmann Interactive Supernova data Repository (Yaron \& Gal-Yam 2012). We considered also the websites of Central Bureau for Astronomical Telegrams ${ }^{5}$, the Astronomer's Telegram $^{6}$, and other references with information on the SN subclasses (e.g. Silverman et al. 2012; Folatelli et al. 2013; Könyves-Tóth et al. 2020). Note that we included in the 91T-like SNe subclass the transitional 99aa-like events. Very early spectra of these $\mathrm{SNe}$ resemble those of 91T-like events. The difference with 91T-like SNe is that in the spectra of 99aa-like $\mathrm{SNe}$ the $\mathrm{Ca}$ II lines are always prominent. However, at maximum light the 99aa-like SNe spectra are similar to those of normal SNe Ia. In addition, the photometry shows that 99aa-like SNe are similarly luminous and slow-declining as 91Tlike $\mathrm{SNe}$. At the same time, we included two transitional 86G-like events in the 91bg-like SNe subclass. Spectroscopically, these events show properties intermediate between normal and 91bg-like SNe. The Si II lines are as strong as in 91bg-like spectra, while Ti II lines are somewhat weaker. The LCs of 86G-like events peak at magnitudes intermediate between those of normal and 91bg-like SNe, and decline rapidly similar to the latter. For more details on the properties of these transitional events, the reader is referred to Taubenberger (2017). In total, we succeeded to collect the subclasses for

\footnotetext{
3 In some cases, the values of $E(B-V)$ towards $\mathrm{SN}$ can reach up to $\sim$ $0.5 \mathrm{mag}$, and only in unique cases up to around $1 \mathrm{mag}$ (e.g. Ashall et al. 2016; Uddin et al. 2020). However, in all these cases the errors in $\Delta m_{15}$ estimations are higher, reaching up to about $0.2 \mathrm{mag}$.

4 The stretch parameter, usually used in cosmological studies for the standardization of SN Ia, is related to the width of the SN Ia LC (see Coelho et al. 2015 , for more details).

5 http://www.cbat.eps.harvard.edu/iau/cbat.html

6 http://www.astronomerstelegram.org/
}

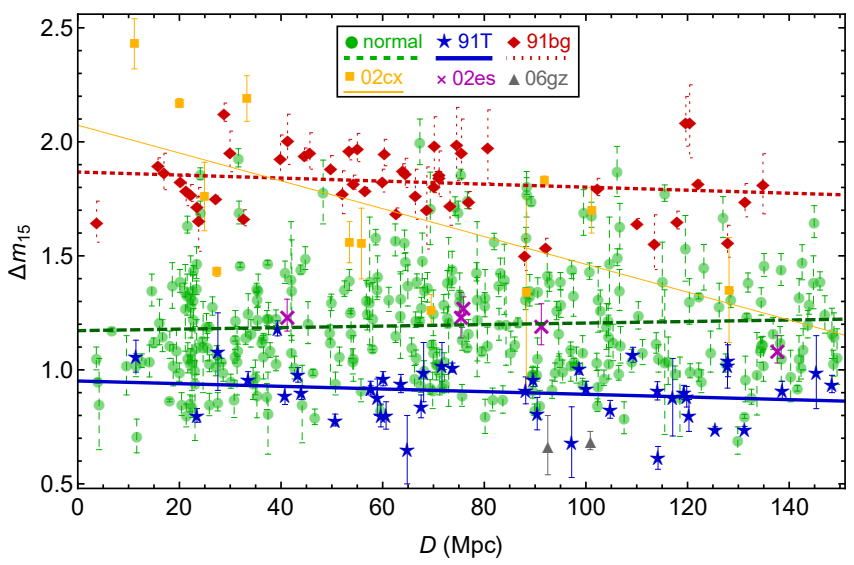

Figure 1. Variation of the $B$-band LC drop, $\Delta m_{15}$, of different subclasses of SNe Ia as a function of the host galaxy distance. Because of very few data points for 02es- and 06gz-like SNe, their best-fitting lines are useless and not presented.

$414 \mathrm{SNe}$ Ia with available LC decline rates. The second column of Table 1 shows the numbers of different subclasses of the collected Type Ia SNe.

We compared the fractions of SN subclasses in our sample with those in the volume-limited sample of the Lick Observatory Supernova Search (LOSS; Li et al. 2011). Based on the detection efficiency of LOSS, the authors noted that their survey did not miss any $\mathrm{SNe}$ Ia that exploded within the sample of targeted galaxies out to $80 \mathrm{Mpc}$, achieving a completeness more than 98 per cent (74 events). Table 1 shows that the representation of the SN subclasses in our sample is in good agreement within errors with that in the LOSS. We checked this by applying the two-sample Kolmogorov-Smirnov (KS) and Anderson-Darling (AD) tests on the reconstructed two discrete data sets (distributions) with sizes of 414 and 74, using the ordered frequencies $\{303,42,50,12,5,2\}$ Our and $\{52,7,11,2,2,0\}_{\text {LOSS }}$ from Table 1 , respectively. The $P$-value of two-sample KS (AD) test represents the probability that the two distributions being compared are randomly drawn from the same parent distribution. Following consolidated tradition, in this paper we adopted the threshold of $P=0.05$ for significance levels of statistical tests. The KS and AD tests showed that the frequencies of SN subclasses in our and LOSS samples are not significantly different $\left(P_{\mathrm{KS}}=0.728\right.$ and $\left.P_{\mathrm{AD}}=0.929\right)$.

For small-size samples like in Table 1, in addition to the original two-sample KS (or AD) test, a Monte Carlo (MC) simulation is usually used for a better approximation of the $P$-value (e.g. Feigelson $\&$ Babu 2012). If the original two data samples have $m$ and $n$ members, the MC simulation randomly generates partitions of joint $m+n$ size sample into an $m$-set and an $n$-set, computing the two-sample KS (or AD) test statistic $D(m, n)$ for the generated sets. The $P_{\mathrm{KS}}^{\mathrm{MC}}$ value (or $P_{\mathrm{AD}}^{\mathrm{MC}}$-value) is the proportion of $D_{i}(m, n)$ values that are greater than $d$, where $i$ is the number of iterations (we used $i=10^{5}$ ) and $d$ is the test statistic based on the original two data samples. The described analysis also showed that the frequencies of SN subclasses in our and LOSS samples are consistent between each other $\left(P_{\mathrm{KS}}^{\mathrm{MC}}=0.710\right.$ and $\left.P_{\mathrm{AD}}^{\mathrm{MC}}=0.650\right)$. Thus, we believe that the artificial loss/excess of any of the SN subclasses in our sample is not significant.

It is important to recall that the 91T-like SNe have peak luminosities that are $\sim 0.6$ mag overluminous than do normal SNe Ia, while 91bg-like SNe have luminosities that are $\sim 2$ mag lower in com- 
Table 2. Numbers of SNe Ia subclasses and coefficients of the linear bestfits $\left(\Delta m_{15}=a+b D[\mathrm{Mpc}]\right)$ from Fig. 1 with results of the Spearman's rank correlation test.

\begin{tabular}{lrcrrc}
\hline SN subclass & $N_{\mathrm{SN}}$ & $a$ & $b \times 10^{-3}$ & \multicolumn{1}{c}{$r_{\mathrm{s}}$} & $P_{\mathrm{S}}$ \\
\hline normal & 303 & $1.17 \pm 0.03$ & $0.3 \pm 0.3$ & 0.057 & 0.320 \\
$91 \mathrm{~T}$ & 42 & $0.95 \pm 0.05$ & $-0.6 \pm 0.5$ & -0.131 & 0.407 \\
$91 \mathrm{bg}$ & 50 & $1.87 \pm 0.04$ & $-0.7 \pm 0.6$ & -0.135 & 0.350 \\
$02 \mathrm{cx}$ & 12 & $2.07 \pm 0.18$ & $-6.1 \pm 2.6$ & -0.559 & 0.058
\end{tabular}

Notes. Spearman's coefficient $\left(r_{\mathrm{s}} \in[-1 ; 1]\right)$ is a nonparametric measure of rank correlation, it assesses how well the relationship between two variables can be described using a monotonic function. Null hypothesis of the test is that the variables are independent, and alternative hypothesis is that they are not $\left(P_{\mathrm{s}} \leqslant 0.05\right)$.

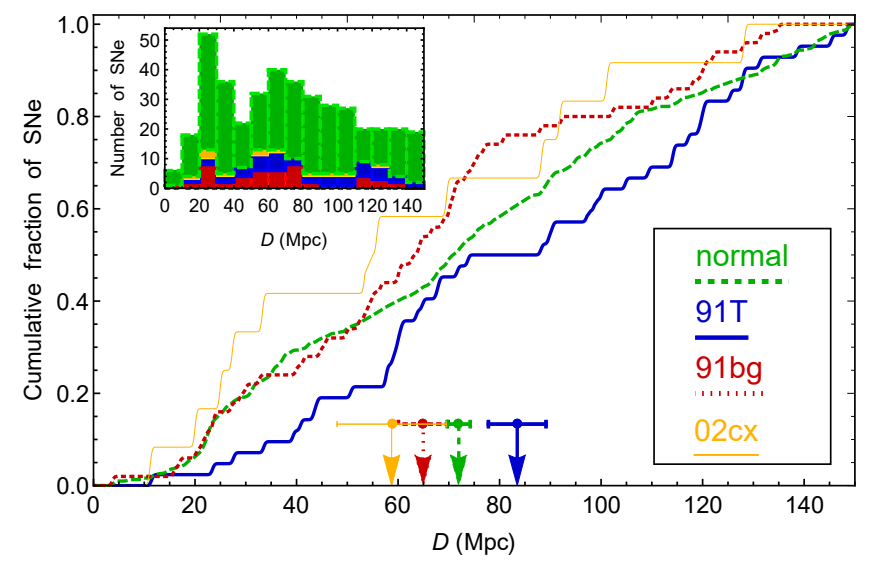

Figure 2. Cumulative distributions and stacked histogram (upper left inset) of distances of the subclasses of Type Ia SNe. The mean values (with standard errors) of the distributions are shown by arrows (with error bars).

parison with normal ones. In general, the peak luminosities of 91bgand 02es-like SNe are comparable. On the other hand, the peak luminosities of some 06gz-like events are even higher than those of 91T-like $\mathrm{SNe}$, while the 02cx-like $\mathrm{SNe}$ can be less luminous than 91bg-like SNe (see Taubenberger 2017, for a review on the extremes of SNe Ia). Therefore, the discoveries of the events with lower luminosities might be complicated at greater distances.

To check the possible influence of the distance effect, in Fig. 1 we illustrated the dependence of extinction-independent $\Delta m_{15}$, which is a good proxy for the intrinsic luminosity of a SN Ia (e.g. Phillips 1993), on distance for the different SN subclasses. The parameters of best-fitting lines from Fig. 1 and results of the Spearman's rank correlation test for $\Delta m_{15}$ versus distance (in Mpc) are presented in Table 2. The Spearman's rank test shows not significant trends between the $\Delta m_{15}$ and distances for all the SN subclasses. Only for $02 \mathrm{cx}$-like $\mathrm{SNe}$, the $P_{\mathrm{s}}$-value of negative trend $\left(r_{\mathrm{s}}<0\right)$ is close to the 0.05 threshold, but still statistically not significant (see Table 2).

Note that, in this study we avoided merging 02cx- and 02es-like SNe subsamples, because they are two distinct subclasses based on their spectroscopic, photometric, and host galaxy properties (e.g. White et al. 2015). For this reason and due to a small number of 02es-like events, we simply removed these five SNe from our analysis. For the same reasons, we also removed two 06gz-like SNe from our statistical/comparative study. These unusual and rare $\mathrm{SNe}$ (02esand 06gz-like) and their host galaxies will be the subject of a forthcoming paper in this series.
Table 3. Comparison of the distributions of distances $D$ (in Mpc) among different $\mathrm{SN}$ subclasses.

\begin{tabular}{lllllllll}
\hline \multicolumn{2}{c}{ SN subsample 1 } & vs & \multicolumn{2}{c}{ SN subsample 2} & $P_{\mathrm{KS}}$ & $P_{\mathrm{AD}}$ & $P_{\mathrm{KS}}^{\mathrm{MC}}$ & $P_{\mathrm{AD}}^{\mathrm{MC}}$ \\
Subclass & $\langle D\rangle$ & & Subclass & $\langle D\rangle$ & & & & \\
\hline normal & $72 \pm 2$ & vs & $91 \mathrm{~T}$ & $83 \pm 6$ & 0.094 & 0.071 & 0.089 & 0.074 \\
normal & $72 \pm 2$ & vs & $91 \mathrm{bg}$ & $65 \pm 5$ & 0.137 & 0.206 & 0.131 & 0.216 \\
normal & $72 \pm 2$ & vs & $02 \mathrm{cx}$ & $59 \pm 11$ & 0.613 & 0.426 & 0.594 & 0.471 \\
$91 \mathrm{~T}$ & $83 \pm 6$ & vs & $91 \mathrm{bg}$ & $65 \pm 5$ & 0.060 & 0.055 & 0.056 & 0.061 \\
$91 \mathrm{~T}$ & $83 \pm 6$ & vs & $02 \mathrm{cx}$ & $59 \pm 11$ & 0.126 & $\mathbf{0 . 0 3 8}$ & 0.104 & 0.051 \\
$91 \mathrm{bg}$ & $65 \pm 5$ & vs & $02 \mathrm{cx}$ & $59 \pm 11$ & 0.863 & 0.843 & 0.822 & 0.867 \\
\hline
\end{tabular}

Notes. The $P_{\mathrm{KS}}$ is the two-sample KS test probability that the two distributions being compared (with respective means and standard errors) are drawn from the same parent distribution. The $P_{\mathrm{KS}}^{\mathrm{MC}}$ is the KS test probability using a MC simulation with $10^{5}$ iterations as explained in the text. Analogically, the $P_{\mathrm{AD}}$ and $P_{\mathrm{AD}}^{\mathrm{MC}}$ probabilities are from the $\mathrm{AD}$ test. The statistically significant difference between the distributions is highlighted in bold.

To further check the redshift-dependent biases in our SN sample, in Fig. 2 we compared the cumulative distance distributions of the SN subclasses. In Table 3, the two-sample KS and AD tests showed that the distance distributions of normal, 91T-, and 91bglike events are not significantly different from one another and could thus be drawn from the same parent distribution. However, only in the AD statistic, the distance distributions of 91T- and 02cx-like SNe are not consistent between each other. At the same time, the corresponding $P$-value for a MC simulation is slightly higher than the significance threshold (see Table 3). Therefore, in comparison with more luminous $\mathrm{SNe}$, the detection of $02 \mathrm{cx}$-like events might be biased/complicated at greater distances (see Fig. 1 and the mean values in Fig. 2), most probably due to their low intrinsic luminosity at maximum light (e.g. Taubenberger 2017). With this in mind, we will be cautious in our comparison of the properties of $02 \mathrm{cx}$-like $\mathrm{SNe}$ and their host galaxies with those of other SN subclasses. None of our SN Ia subsamples (except perhaps $02 \mathrm{cx}$-like) should be affected by redshift-dependent biases.

\subsection{SN Ia host galaxy sample}

Our sample of SN Ia host galaxies was obtained by cross-matching the coordinates of our $407 \mathrm{SNe}$ Ia with the footprints of the SDSS sixteenth data release (DR16; Ahumada et al. 2020), the PanSTARRS second data release (DR2; Chambers et al. 2016), and the SkyMapper second data release (DR2; Onken et al. 2019), using the techniques described in Hakobyan et al. (2012). Note that these surveys cover together the entire sky, complementing one another, and with some intersection. As a result, we identified 394 individual host galaxies of all collected SNe Ia: 1, 2, and $4 \mathrm{SNe}$ are found in 383, 10 , and 1 galaxies, respectively. It should be noted that some of the identified host galaxies from the footprint of SDSS DR16 are already listed in databases of Hakobyan et al. (2012, 2016, 2017); Karapetyan et al. (2018); Barkhudaryan et al. (2019), which are based on older data releases of SDSS. We nevertheless re-implemented the entire reduction process for all SDSS host galaxies using the DR16 properties.

Following the approach described in detail in Hakobyan et al. (2012), we morphologically classified all 394 host galaxies by visual 
Table 4. Numbers of the subclasses of Type Ia SNe at distances $\leqslant 150 \mathrm{Mpc}$ as a function of morphological types of host galaxies.

\begin{tabular}{lrrrrrrrrrrrrrr}
\hline & E & E/S0 & S0 & S0/a & Sa & Sab & Sb & Sbc & Sc & Scd & Sd & Sdm & Sm & All \\
\hline normal & 20 & 7 & 20 & 22 & 20 & 15 & 57 & 63 & 47 & 13 & 8 & 7 & 4 & 303 \\
$91 \mathrm{~T}$ & 0 & 0 & 1 & 1 & 1 & 5 & 7 & 13 & 4 & 4 & 2 & 2 & 2 & 42 \\
$91 \mathrm{bg}$ & 17 & 3 & 3 & 8 & 5 & 5 & 1 & 4 & 4 & 0 & 0 & 0 & 0 & 50 \\
$02 \mathrm{cx}$ & 0 & 0 & 0 & 1 & 0 & 0 & 2 & 3 & 2 & 1 & 0 & 2 & 1 & 12 \\
All & 37 & 10 & 24 & 32 & 26 & 25 & 67 & 83 & 57 & 18 & 10 & 11 & 7 & 407 \\
\hline
\end{tabular}

inspection of images of hosts from the $\mathrm{SDSS}^{7}$, Pan-STARRS ${ }^{8}$, and SkyMapper ${ }^{9}$ imaging servers, all of which build RGB colour images from the $g, r$, and $i$ data channels. Note that SDSS, Pan-STARRS, and SkyMapper use different ugriz, grizy, and uvgriz filters, respectively. However, the gri sets of Pan-STARRS and SkyMapper are very similar to the SDSS filters of the same names (Chambers et al. 2016; Wolf et al. 2018). The SDSS RGB colour images of typical examples of SNe host galaxies with morphological classification according to the modified Hubble sequence (E-E/S0-SO-SO/a-SaSab-Sb-Sbc-Sc-Scd-Sd-Sdm-Sm) can be found in Hakobyan et al. (2012). Table 4 displays the distributions of the subclasses of SNe Ia among the various morphological types of host galaxies.

We first applied corrections to transform the Pan-STARRS and SkyMapper photometry to the SDSS system (see Finkbeiner et al. 2016; Wolf et al. 2018; Onken et al. 2019). For all host galaxies, we fitted $25 \mathrm{mag} \operatorname{arcsec}^{-2}$ elliptical apertures in the SDSS $g$-band according to the approach presented in Hakobyan et al. (2012). Following Barkhudaryan et al. (2019), for each host galaxy the corresponding SDSS ugriz fluxes (apparent magnitudes ${ }^{10}$ ) are measured using the mentioned elliptical apertures in $g$-band. The $u$-band flux measurements are performed only for host galaxies located on the footprints of SDSS and SkyMapper survey, which have the corresponding filter support. During the flux measurements, we masked out bright projected/saturated stars. The absolute/apparent magnitudes are corrected for Galactic extinction based on the Schlafly \& Finkbeiner (2011) recalibration of the Schlegel, Finkbeiner \& Davis (1998) dust map. In addition, these values are corrected for elongation/inclination effects and for the host galaxy internal extinction according to Bottinelli et al. (1995). Finally, the colour-based Kcorrections (Chilingarian, Melchior \& Zolotukhin 2010) are mostly negligible $(<0.15 \mathrm{mag})$, since the host redshifts are $\lesssim 0.036$.

The database of 407 individual SNe Ia (SN designation, spectroscopic subclass, $\Delta m_{15}$, and corresponding sources of the data) and their 394 hosts (galaxy designation, distance, corrected ugriz apparent magnitudes, and morphological type) is available in the online version (Supporting Information) of this article.

\section{RESULTS AND DISCUSSION}

With the aim of clarifying the progenitor natures of normal and peculiar (91T-, 91bg-, and 02cx-like) SN Ia subclasses, in this section

\footnotetext{
7 http://skyserver.sdss.org/dr16/en/tools/chart/listinfo.aspx

8 https://ps1images.stsci.edu/cgi-bin/ps1cutouts

9 http://skymapper.anu.edu.au/sky-viewer/

10 The magnitudes are in the $\mathrm{AB}$ system (for more details, see https://www.sdss.org/dr16/algorithms/fluxcal/).
}
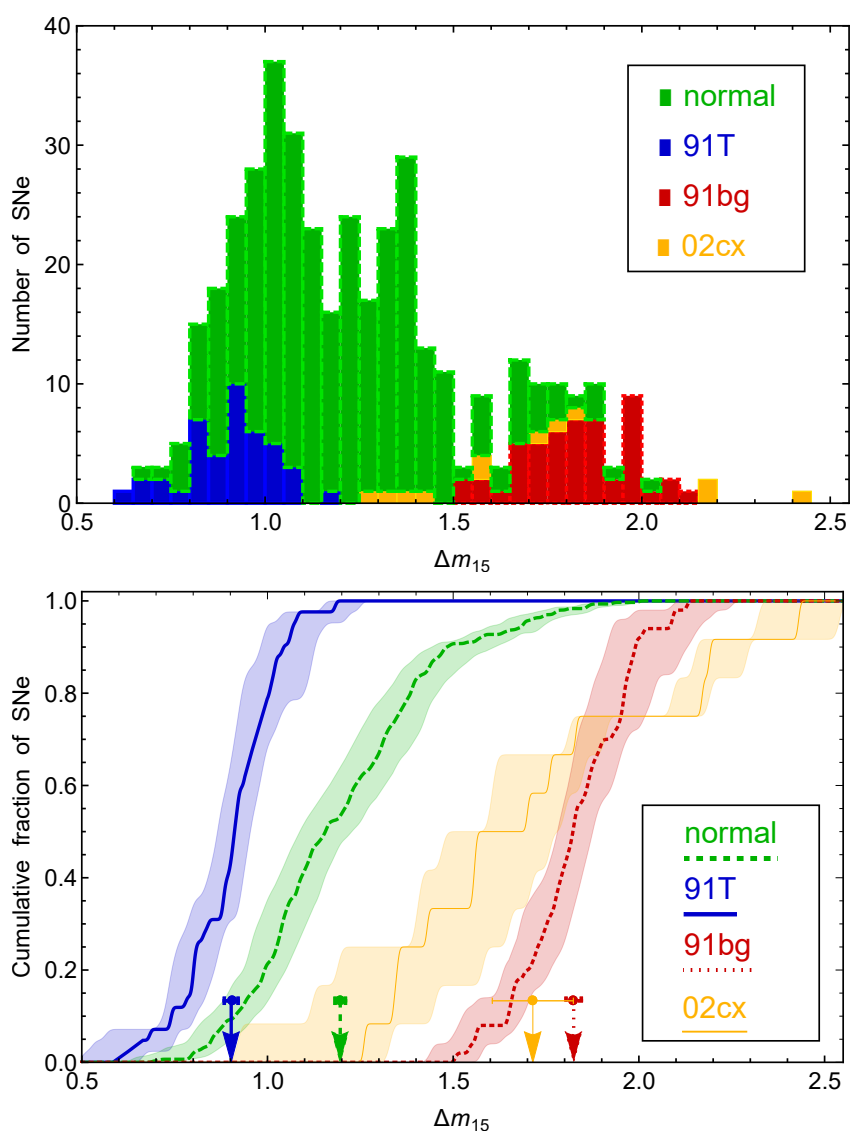

Figure 3. Upper panel: stacked histogram of the $B$-band $\Delta m_{15}$ values of different SN Ia subclasses. Bottom panel: cumulative $\Delta m_{15}$ distributions for the SN subclasses. The light shaded regions around each cumulative curve show the corresponding spreads considering the uncertainties in $\Delta m_{15}$ values. The mean values (with standard errors) of the distributions are shown by arrows (with error bars).

we comparatively study the important relations between the LC decline rates of these SNe Ia and the global properties of the stellar population of their host galaxies with different morphological types.

\subsection{Light curve decline rates of SN Ia subclasses}

Fig. 3 shows the histogram and cumulative distributions of $B$-band $\Delta m_{15}$ values for the different SN Ia subclasses in our sample. The $\Delta m_{15}$ distribution appears to be bimodal, with the second (weaker) mode mostly distributed within $\sim 1.5-2.1$ mag range. This faster declining SN range is dominated by $91 \mathrm{bg}$-like (subluminous) events, while the $\Delta m_{15}$ of 91T-like (overluminous) SNe are distributed only within the first mode at slower declining range with $\Delta m_{15} \lesssim 1.1 \mathrm{mag}$. This picture of the $\Delta m_{15}$ distributions of faster and slower declining Type Ia SNe is very similar to the $\Delta m_{15}$ distribution found by Ashall et al. (2016), who studied a data set of 165 low redshift $(z<0.06)$ SNe Ia (see also Pakmor et al. 2013; Siebert et al. 2019). It should be noted that the separate ranges of $\Delta m_{15}$ distributions of 91T- and 91bg-like $\mathrm{SNe}$ are almost equal to each other $(\sim 0.6 \mathrm{mag})$, but about 2.2 times narrower than that of normal SNe Ia $(\sim 1.3 \mathrm{mag})$. At the same time, despite the small number statistics of $02 \mathrm{cx}$-like $\mathrm{SNe}$, their $B$-band decline rates with some extremes are spread in the faster side of the $\Delta m_{15}$ distribu- 
Table 5. Comparison of the $B$-band $\Delta m_{15}$ distributions among different SN Ia subclasses.

\begin{tabular}{|c|c|c|c|c|c|c|c|c|}
\hline \multicolumn{2}{|c|}{ SN subsample 1} & \multirow[t]{2}{*}{ vs } & \multicolumn{2}{|c|}{ SN subsample 2} & \multirow[t]{2}{*}{$P_{\mathrm{KS}}$} & \multirow[t]{2}{*}{$P_{\mathrm{AD}}$} & \multirow[t]{2}{*}{$P_{\mathrm{KS}}^{\mathrm{MC}}$} & \multirow[t]{2}{*}{$P_{\mathrm{AD}}^{\mathrm{MC}}$} \\
\hline Subclass & $\left\langle\Delta m_{15}\right\rangle$ & & Subclass & $\left\langle\Delta m_{15}\right\rangle$ & & & & \\
\hline normal & $1.19 \pm 0.01$ & vs & $91 \mathrm{~T}$ & $0.90 \pm 0.02$ & $<0.001$ & $<0.001$ & $<0.001$ & $<\mathbf{0 . 0 0 1}$ \\
\hline normal & $1.19 \pm 0.01$ & vs & $91 \mathrm{bg}$ & $1.82 \pm 0.02$ & $<0.001$ & $<0.001$ & $<0.001$ & $<\mathbf{0 . 0 0 1}$ \\
\hline normal & $1.19 \pm 0.01$ & vs & $02 \mathrm{cx}$ & $1.71 \pm 0.11$ & $<0.001$ & $<0.001$ & $<0.001$ & $<\mathbf{0 . 0 0 1}$ \\
\hline $91 \mathrm{~T}$ & $0.90 \pm 0.02$ & vs & $91 \mathrm{bg}$ & $1.82 \pm 0.02$ & $<\mathbf{0 . 0 0 1}$ & $<0.001$ & $<0.001$ & $<0.001$ \\
\hline $91 \mathrm{~T}$ & $0.90 \pm 0.02$ & vs & $02 \mathrm{cx}$ & $1.71 \pm 0.11$ & $<\mathbf{0 . 0 0 1}$ & $<\mathbf{0 . 0 0 1}$ & $<\mathbf{0 . 0 0 1}$ & $<\mathbf{0 . 0 0 1}$ \\
\hline $91 \mathrm{bg}$ & $1.82 \pm 0.02$ & vs & $02 \mathrm{cx}$ & $1.71 \pm 0.11$ & 0.031 & 0.002 & 0.025 & 0.003 \\
\hline
\end{tabular}

Notes. The explanations for $P$-values are the same as in Table 3 . The statistically significant differences between the distributions are highlighted in bold.

tion of normal SNe Ia (see also Stritzinger et al. 2015). The range of $\Delta m_{15}$ distribution of these peculiar events is $\sim 1.2 \mathrm{mag}$.

In Table 5, two-sample KS and AD tests show that the $\Delta m_{15}$ distributions are inconsistent significantly between any pairs of SN Ia subclasses of our sample. Therefore, the $\Delta m_{15}$ distributions of 91Tand $91 \mathrm{bg}$-like $\mathrm{SNe}$, which cross the tails of $\Delta m_{15}$ distribution of normal SNe Ia, without crossing one another (see Fig. 3), suggest that these SN Ia subclasses may come from different stellar populations (e.g. Pakmor et al. 2013; Ashall et al. 2016). A similar idea can be viable also for the progenitor population of 02cx-like $\mathrm{SNe}$ (see Jha 2017, and references therein), when comparing the $\Delta m_{15}$ properties of normal SNe Ia with those of 02cx-like events (Fig. 3 and Table 5).

In next subsections, we obtain more robust constraints on the SN Ia progenitor populations, by studying the host galaxy global properties combined to the distributions of LC decline rates of the SN subclasses.

\subsection{Morphologies of host galaxies of SN Ia subclasses}

The upper panel of Fig. 4 presents the cumulative distributions of host galaxy morphological types of the subclasses of SNe Ia. It is clear that the host galaxies of normal, 91T- (overluminous), and 91bg-like (subluminous) SNe Ia have morphological type distributions that are significantly mutually inconsistent (see Table 6). Host galaxies of $91 \mathrm{bg}$-like $\mathrm{SNe}$ have, on average, earlier morphological types $(\langle t\rangle \approx 0)$, with 46 per cent of the events discovered in E-S0 galaxies. In contrast, host galaxies of 91T-like SNe have, on average, later morphological types $(\langle t\rangle \approx 4)$, with a single 91T-like event in E-S0 hosts (only $\sim 2$ per cent of the subsample, see Table 4 ). The morphological distribution of host galaxies of spectroscopically normal SNe Ia occupies an intermediate position between the host morphologies of 91T- and 91bg-like events. The morphological distribution of 02cx-like SNe hosts is similar to that of 91T-like SNe hosts, though the LC decline rates of the former SN subclass are significantly larger than those of the latter subclass (see Fig. 3 and Table 5).

These results are in good agreement with those of GonzálezGaitán et al. (2014), who presented a photometric identification technique for $91 \mathrm{bg}$-like $\mathrm{SNe}$, separating them from the normal Type Ia population, and comparatively studied SNe host galaxy morphologies (see also Foley et al. 2009; Valenti et al. 2009; Perets et al. 2010, for other SN Ia subclasses). González-Gaitán et al. showed that the morphological distribution of host galaxies of $91 \mathrm{bg}$-like candidates is significantly earlier $\left(P_{\mathrm{KS}} \simeq 0.002\right)$ from that of normal SNe Ia. ${ }^{11}$
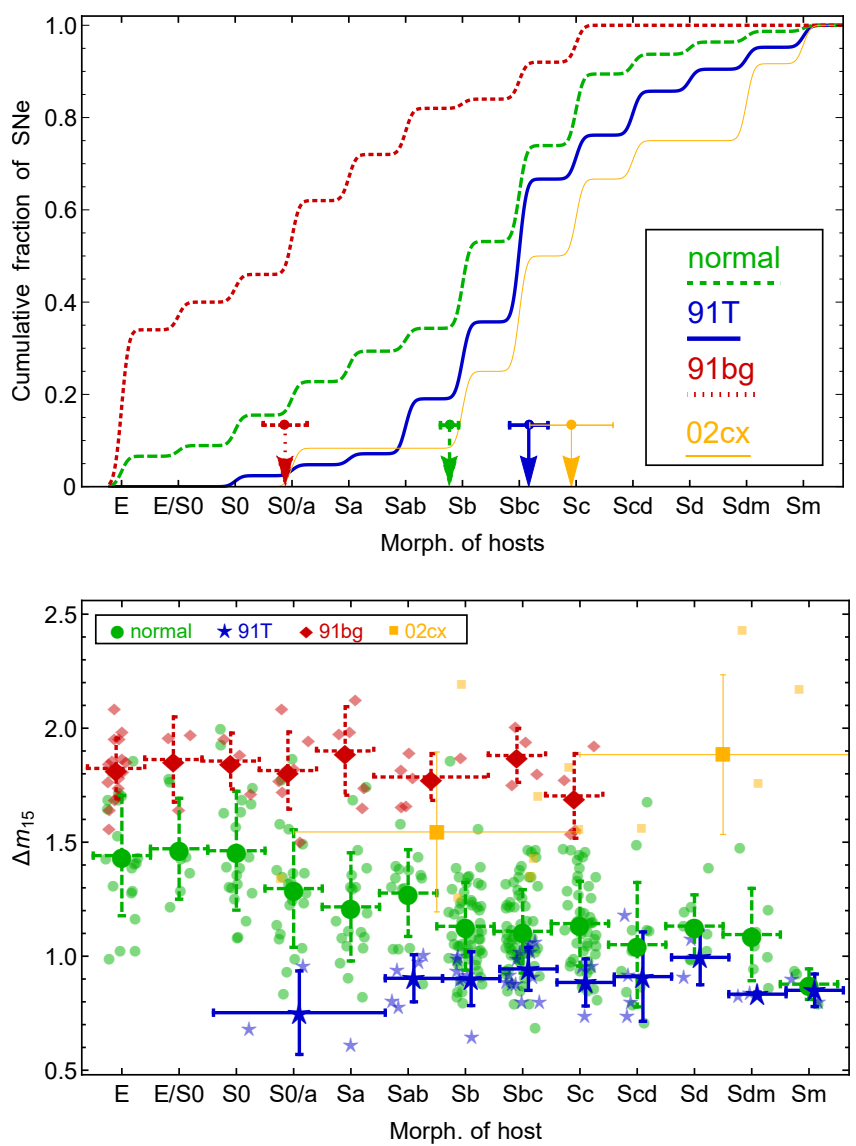

Figure 4. Upper panel: cumulative distributions of host galaxy morphological types of the subclasses of Type Ia SNe. The mean morphological types (with standard errors) of the host subsamples are shown by arrows (with error bars). Bottom panel: $B$-band $\Delta m_{15}$ versus host galaxy morphology for different $\mathrm{SNe}$ Ia subclasses, displayed as a scatter plot (smaller symbols) and averaged in bins of morphological type (bigger symbols). The abscissae of the smaller symbols are randomly shifted by \pm 0.3 in $t$ for clarity, while they are slightly shifted according to SN Ia subclass for the bigger symbols. The S0, S0/a, and Sa morphological bins of 91T-like SNe are merged because each of them contains only one 91T-like event. For 91bg-like SNe, the Sb bin contains only one SN, therefore it is merged with the Sab bin. Similarly, given their small number, the hosts of $02 \mathrm{cx}$-like $\mathrm{SNe}$ are merged into two broad morphological bins. 
Table 6. Comparison of the distributions of host galaxy morphological types among different SN Ia subclasses.

\begin{tabular}{|c|c|c|c|c|c|c|c|c|}
\hline \multicolumn{2}{|c|}{ Host subsample 1} & \multirow[t]{2}{*}{ vs } & \multicolumn{2}{|c|}{ Host subsample 2} & \multirow[t]{2}{*}{$P_{\mathrm{KS}}$} & \multirow[t]{2}{*}{$P_{\mathrm{AD}}$} & \multirow[t]{2}{*}{$P_{\mathrm{KS}}^{\mathrm{MC}}$} & \multirow[t]{2}{*}{$P_{\mathrm{AD}}^{\mathrm{MC}}$} \\
\hline SN subclass & $\langle t\rangle$-type & & SN subclass & $\langle t\rangle$-type & & & & \\
\hline normal & $2.8 \pm 0.1$ & vs & $91 \mathrm{~T}$ & $4.2 \pm 0.3$ & 0.015 & 0.004 & 0.015 & 0.007 \\
\hline normal & $2.8 \pm 0.1$ & vs & $91 \mathrm{bg}$ & $-0.1 \pm 0.4$ & $<\mathbf{0 . 0 0 1}$ & $<0.001$ & $<\mathbf{0 . 0 0 1}$ & $<\mathbf{0 . 0 0 1}$ \\
\hline normal & $2.8 \pm 0.1$ & vs & $02 \mathrm{cx}$ & $4.9 \pm 0.7$ & 0.134 & 0.006 & 0.122 & 0.015 \\
\hline $91 \mathrm{~T}$ & $4.2 \pm 0.3$ & vs & $91 \mathrm{bg}$ & $-0.1 \pm 0.4$ & $<\mathbf{0 . 0 0 1}$ & $<0.001$ & $<\mathbf{0 . 0 0 1}$ & $<\mathbf{0 . 0 0 1}$ \\
\hline $91 \mathrm{~T}$ & $4.2 \pm 0.3$ & vs & $02 \mathrm{cx}$ & $4.9 \pm 0.7$ & 0.673 & 0.470 & 0.668 & 0.475 \\
\hline $91 \mathrm{bg}$ & $-0.1 \pm 0.4$ & vs & $02 \mathrm{cx}$ & $4.9 \pm 0.7$ & $<\mathbf{0 . 0 0 1}$ & $<0.001$ & $<\mathbf{0 . 0 0 1}$ & $<\mathbf{0 . 0 0 1}$ \\
\hline
\end{tabular}

Notes. The explanations for $P$-values are the same as in Table 3. The statistically significant differences between the distributions are highlighted in bold.

Table 7. Comparison of the $B$-band $\Delta m_{15}$ distributions of SNe Ia among different subsamples of host morphologies.

\begin{tabular}{|c|c|c|c|c|c|c|c|c|c|c|c|c|}
\hline \multirow[b]{2}{*}{ Host } & \multicolumn{2}{|c|}{ Subsample 1} & \multirow[b]{2}{*}{$\left\langle\Delta m_{15}\right\rangle$} & \multirow[t]{2}{*}{ vs } & \multirow{2}{*}{ Host } & \multicolumn{2}{|c|}{ Subsample 2} & \multirow[b]{2}{*}{$\left\langle\Delta m_{15}\right\rangle$} & \multirow[t]{2}{*}{$P_{\mathrm{KS}}$} & \multirow[t]{2}{*}{$P_{\mathrm{AD}}$} & \multirow[t]{2}{*}{$P_{\mathrm{KS}}^{\mathrm{MC}}$} & \multirow[t]{2}{*}{$P_{\mathrm{AD}}^{\mathrm{MC}}$} \\
\hline & SN subclass & $N_{\mathrm{SN}}$ & & & & SN subclass & $N_{\mathrm{SN}}$ & & & & & \\
\hline E-S0 & normal & 47 & $1.45 \pm 0.04$ & vs & $\mathrm{S} 0 / \mathrm{a}-\mathrm{Sm}$ & normal & 256 & $1.15 \pm 0.01$ & $<\mathbf{0 . 0 0 1}$ & $<\mathbf{0 . 0 0 1}$ & $<0.001$ & $<0.001$ \\
\hline E-S0 & $91 \mathrm{bg}$ & 23 & $1.83 \pm 0.03$ & vs & $\mathrm{S} 0 / \mathrm{a}-\mathrm{Sm}$ & $91 \mathrm{bg}$ & 27 & $1.82 \pm 0.03$ & 0.876 & 0.840 & 0.806 & 0.842 \\
\hline E-S0 & normal & 47 & $1.45 \pm 0.04$ & vs & $\mathrm{S} 0 / \mathrm{a}-\mathrm{Sbc}$ & normal & 177 & $1.16 \pm 0.02$ & $<\mathbf{0 . 0 0 1}$ & $<0.001$ & $<\mathbf{0 . 0 0 1}$ & $<\mathbf{0 . 0 0 1}$ \\
\hline E-S0 & normal & 47 & $1.45 \pm 0.04$ & vs & $\mathrm{Sc}-\mathrm{Sm}$ & normal & 79 & $1.11 \pm 0.02$ & $<\mathbf{0 . 0 0 1}$ & $<\mathbf{0 . 0 0 1}$ & $<\mathbf{0 . 0 0 1}$ & $<\mathbf{0 . 0 0 1}$ \\
\hline $\mathrm{S} 0 / \mathrm{a}-\mathrm{Sbc}$ & normal & 177 & $1.16 \pm 0.02$ & vs & $\mathrm{Sc}-\mathrm{Sm}$ & normal & 79 & $1.11 \pm 0.02$ & 0.050 & 0.067 & 0.048 & 0.068 \\
\hline $\mathrm{S} 0 / \mathrm{a}-\mathrm{Sbc}$ & $91 \mathrm{~T}$ & 27 & $0.91 \pm 0.02$ & vs & $\mathrm{Sc}-\mathrm{Sm}$ & $91 \mathrm{~T}$ & 14 & $0.89 \pm 0.03$ & 0.608 & 0.322 & 0.557 & 0.366 \\
\hline E-S0 & $91 \mathrm{bg}$ & 23 & $1.83 \pm 0.03$ & vs & $\mathrm{S} 0 / \mathrm{a}-\mathrm{Sbc}$ & $91 \mathrm{bg}$ & 23 & $1.84 \pm 0.03$ & 0.991 & 0.936 & 0.931 & 0.937 \\
\hline
\end{tabular}

Notes. The explanations for $P$-values are the same as in Table 3 . The statistically significant differences between the distributions are highlighted in bold. Due to small number statistics of $02 \mathrm{cx}$-like SNe, their study in the subsamples of host morphology is statistically useless, and therefore not presented.

In addition, using a Fisher exact test, González-Gaitán et al. noted that the number ratio of normal SNe Ia in passive (E-S0) to starforming ( $\mathrm{S} 0 / \mathrm{a}-\mathrm{Sm})$ galaxies is statistically different (smaller) from the same ratio of $91 \mathrm{bg}$-like $\mathrm{SNe}$ with a probability $P_{\mathrm{F}} \simeq 0.002$. For our SN sample in Table 4, the behaviour of the ratios is the same as in González-Gaitán et al. (2014), with $P_{\mathrm{F}}<0.001$. For the hosts of other SN Ia subclasses, Foley et al. (2009) and Perets et al. (2010) mentioned that 91T- and 02cx-like objects have consistent host morphologies, while hosts of 91T-like SNe have later morphological types in comparison with normal SNe Ia.

Taking into account that the mean stellar population age of galaxies is steadily decreasing along the Hubble sequence from early- to late-type galaxies (e.g. González Delgado et al. 2015), the results in the upper panel of Fig. 4 and Table 6 indicate that the progenitor population age of SN Ia subclasses in the sequence of $91 \mathrm{bg}-$, normal, and 91T(or 02cx)-like events is decreasing as well.

In the bottom panel of Fig. 4, we show the distribution of the $B$-band $\Delta m_{15}$ values as a function of morphological type of host galaxies, for different SN Ia subclasses. When dividing SNe Ia hosts between E-S0 (galaxies with only old stellar component) and S0/aSm morphological types (galaxies with both old and young stellar components), Table 7 shows that the distributions of $\Delta m_{15}$ values of 91bg-like $\mathrm{SNe}$ are not different between the host subsamples, being distributed mainly within old ellipticals/lenticulars and early-type

peculiar SNe Ia subgroup, which includes 02cx- and 06gz-like events. However, the results concerning to the hosts morphologies of this peculiar subgroup are not comparable with those of our case, because their subgroup include nearly equal numbers of 02cx- and 06gz-like SNe whose hosts morphologies have different distributions (e.g. Valenti et al. 2009; Taubenberger et al. 2011; Barkhudaryan et al. 2019), thus mixing the morphological types. spirals (Fig. 4). In contrast, the $\Delta m_{15}$ distribution of normal SNe Ia in $\mathrm{E}-\mathrm{S} 0$ hosts is not consistent with that in $\mathrm{S} 0 / \mathrm{a}-\mathrm{Sm}$ galaxies.

Using narrow morphological bins, i.e. E-S0, S0/a-Sbc, and Sc$\mathrm{Sm}$, shows that the $\Delta m_{15}$ values of normal SNe Ia are decreasing on average from early- to late-type galaxies. Indeed, the Spearman's rank test shows that the significant correlation between $\Delta m_{15}$ and host $t$-types exists only for normal SNe Ia $\left(r_{\mathrm{s}}=-0.416\right.$, $\left.P_{\mathrm{s}}<0.001\right)$, although the discrete $t$-type values are not convenient for the test. The $\Delta m_{15}$ distributions of 91T-like events are not different in S0/a-Sbc and Sc-Sm galaxies (Table 7), which lie mostly within spiral hosts (Fig. 4). Without a clear separation between the SN Ia spectroscopic subclasses, similar results have been obtained in the past with samples of SNe Ia and their host galaxies at different redshifts (e.g. Hamuy et al. 1996, 2000; Howell 2001; Altavilla et al. 2004; Gallagher et al. 2005; Ashall et al. 2016; Pruzhinskaya et al. 2020). Note that, because of few data points for 02cx-like SNe, their study in the subsamples of host morphology is statistically useless, and therefore not presented in Table 7.

Recall that the intrinsic ranges of $\Delta m_{15}$ distributions of 91T- and 91bg-like SNe are narrower than that of normal SNe Ia (see Subsection 3.1). Could these narrow ranges of $\Delta m_{15}$ prevent us from seeing any trends with host galaxy properties such as morphology? We probe this issue by searching for trends of $\Delta m_{15}$ and morphology for normal SNe Ia, in the corresponding narrow ranges of $\Delta m_{15}$ as for 91T-like events first and then as for 91bg-like SNe. For 189 normal SNe Ia with $\Delta m_{15} \lesssim 1.26 \mathrm{mag}$, the $r_{\mathrm{s}}=-0.177$ and $P_{\mathrm{s}}=0.015$, and for 64 normal events with $\Delta m_{15} \gtrsim 1.38 \mathrm{mag}$, the $r_{\mathrm{s}}=-0.305$ and $P_{\mathrm{s}}=0.014$. Thus, normal SNe Ia keep the $\Delta m_{15}$ - morphology trend direction and significance even in each of the narrower $\Delta m_{15}$ ranges, showing that the $\Delta m_{15}$ ranges of 91T- and $91 \mathrm{bg}$-like $\mathrm{SNe}$ should not play a significant role in the absence of the trends for peculiar events. 
Along with the mean stellar population ages of galaxies, the ratio of bulge luminosity (old halo/bulge component) over disc luminosity (old and young star-forming disc components) is steadily decreasing along the Hubble sequence (e.g. Oohama et al. 2009; de Lapparent et al. 2011). There is no disc component in elliptical galaxies, while the stellar bulge component is negligible in late-type spiral galaxies where the star-forming disc is prominent. Therefore, most probably the results above indicate that $91 \mathrm{bg}$-like $\mathrm{SNe}$ (subluminous SNe Ia) come only from the old stellar component (halo/bulge, old disc) of hosts, while 91T-like events (overluminous SNe Ia) originate only from the young component (star-forming disc) of galaxies. Faster and slower declining normal SNe Ia likely come from older and younger stellar populations of host galaxies, respectively. Despite their small numbers, $02 \mathrm{cx}$-like SNe typically lie in star-forming galaxies with late-type morphology (Fig. 4), hinting that these events likely originate from young stellar component.

It is instructive to consider counter-examples. The discovery of SN 2004br (Graham \& Foley 2004), a 91T-like event (Silverman et al. 2012), in NGC 4493 of S0 morphology (see Table 4 and Fig. 4), which consists of only old stellar population from a naive point of view. In NGC 4493, another SN Ia was also discovered (SN 1994M; Wild 1994) with a normal spectroscopic classification (Blondin et al. 2012). Interestingly, this host galaxy has a distorted stellar disc and shows obvious evidence of an interaction with neighbor/companion galaxy (Hakobyan et al. 2012). The age of the younger stellar component in this galaxy is estimated to be down to about a few hundred Myr (Schawinski 2009). There are many indications that residual star formation episodes (birth of relatively young stellar component) could also take place in elliptical or lenticular galaxies, due to galaxy-galaxy interaction with close neighbors (e.g. Kaviraj et al. 2009; Schawinski et al. 2014; Gomes et al. 2016; Ge et al. 2020). In such early-type galaxies, in very rare cases, even core-collapse SNe were discovered (e.g. Hakobyan et al. 2008, 2012; Suh et al. 2011; Lee et al. 2019) whose progenitor ages are thought to be up to about hundred Myr (e.g. Zapartas et al. 2017). Therefore, in our sample, the presence of unique 91T-like SN in the mentioned interacting lenticular galaxy can be interpreted as a result of such a residual star formation that could deliver SN Ia from relatively young progenitors.

\subsection{Colour-mass diagram of SNe Ia host galaxies}

Galaxy colours represent a more quantitative measure of galaxy classes, albeit spectral classes. Therefore, in this subsection we only use the photometric data of SNe Ia hosts, accompanied with the values of SN LC decline rates. To analyse the distribution of SN host galaxies on the colour-mass diagram, we estimate the stellar masses $\left(M_{*}\right)$ of our sample galaxies, using a simple empirical relation of Taylor et al. (2011) between $\log \left(M_{*} / \mathrm{M}_{\odot}\right), g-i$ colour and $i$-band absolute magnitude, $M_{i}$ (see Barkhudaryan et al. 2019, for details). While this mass estimate is rudimentary and may be biased relative to more refined mass measurements, it suffices for our aim to. In the colour-mass diagram (Fig. 5), we prefer to use $u-r$ colours, which present the largest contrast among optical colours between the inputs of young and old stellar populations (e.g. Schawinski et al. 2014; Barkhudaryan et al. 2019). Recall that $u$-band measurements are available only for 326 SNe Ia host galaxies ( 80 per cent of the sample) located in the footprints of the SDSS and SkyMapper survey, which provide the corresponding fits images (see Subsection 2.2).

In the upper panel of Fig. 5, the $u-r$ colour-mass diagram clearly displays a bimodal distribution of colours of SNe Ia host galaxies (see also Perley et al. 2020, for the $g-i$ colours), as seen in general
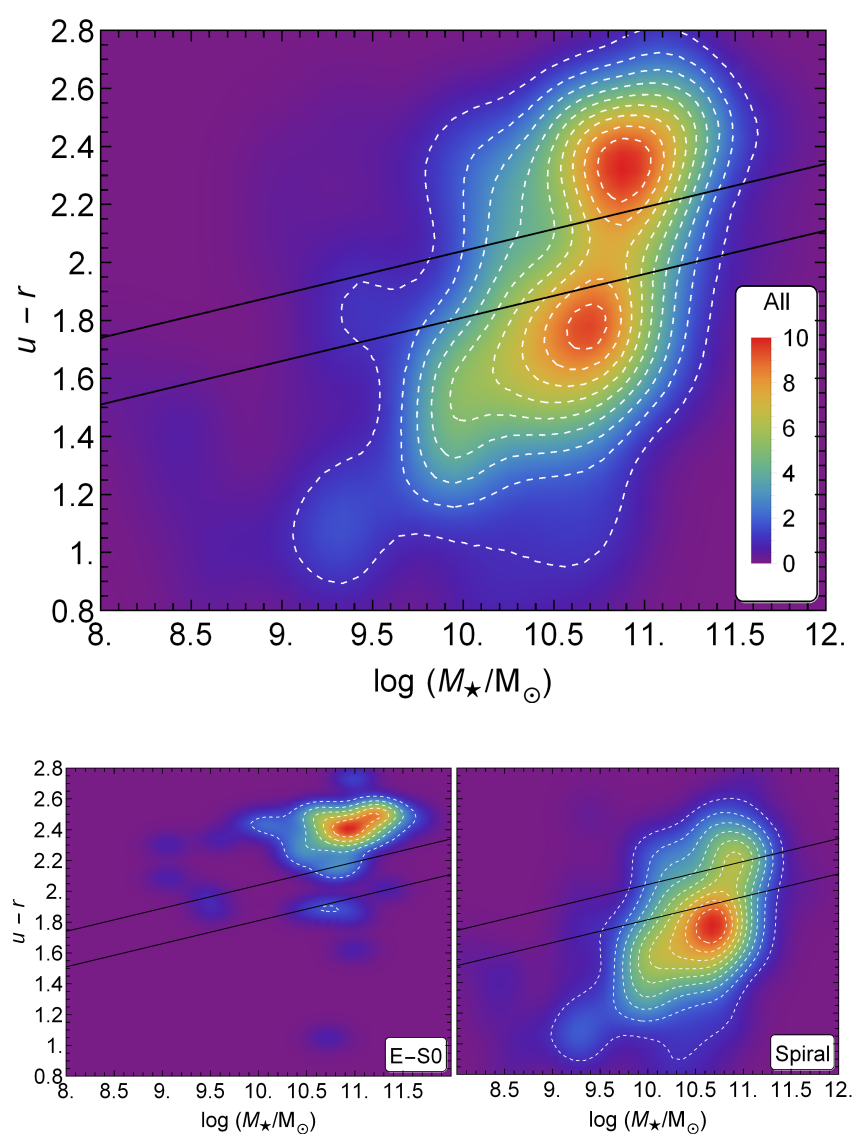

Figure 5. Colour-mass diagrams of SNe Ia host galaxies viewed as density and contours. The region between two solid lines marks the Green Valley (see text for details). Upper panel: all $326 \mathrm{SNe}$ Ia host galaxies with measured $u$ and $r$ magnitudes. The colour bar shows the linear (arbitrary) units of density. Bottom panels: same for the E-S0 (left) and spiral (right) host morphologies.

galaxy samples (e.g. Baldry et al. 2006). More precisely, galaxies with dominant old stellar populations and low specific SFRs (mostly massive galaxies) lie in the so-called Red Sequence of the diagram, which is located at $u-r \gtrsim 2 \mathrm{mag}$, with a tail of about 10 per cent of the population reaching down to $u-r \approx 1.5 \mathrm{mag}$ (e.g. Baldry et al. 2006; Schawinski et al. 2014). Star-forming galaxies with prominent young stellar population are located in the so-called Blue Cloud of the diagram, mostly at $u-r \lesssim 2 \mathrm{mag}$, with a tail to redder colours.

The bottom left panel of Fig. 5 shows the colour-mass relation for our E-S0 hosts with 83 SNe Ia. Similarly, spiral galaxies of the sample that host $243 \mathrm{SNe}$ Ia are presented in the bottom right panel of Fig. 5. The bimodality in the diagram is due to the superposition of colours of these two distinct populations of galaxies (e.g. Schawinski et al. 2014). A dip between bimodal (red and blue) colours in the colour-mass diagram is called Green Valley (the region between two solid lines in Fig. 5, see also Barkhudaryan et al. 2019). This region is thought to include galaxies that are in transitional stage of the evolution between star-forming galaxies in the Blue Cloud and passively evolving quenched galaxies in the Red Sequence (e.g. Baldry et al. 2006; Mendez et al. 2011; Schawinski et al. 2014; McIntosh et al. 2014).

Fig. 6 shows how the host galaxies of different SN Ia subclasses are distributed in the colour-mass diagram. The figure also displays the distributions of $u-r$ colours and masses for host galaxies for the 


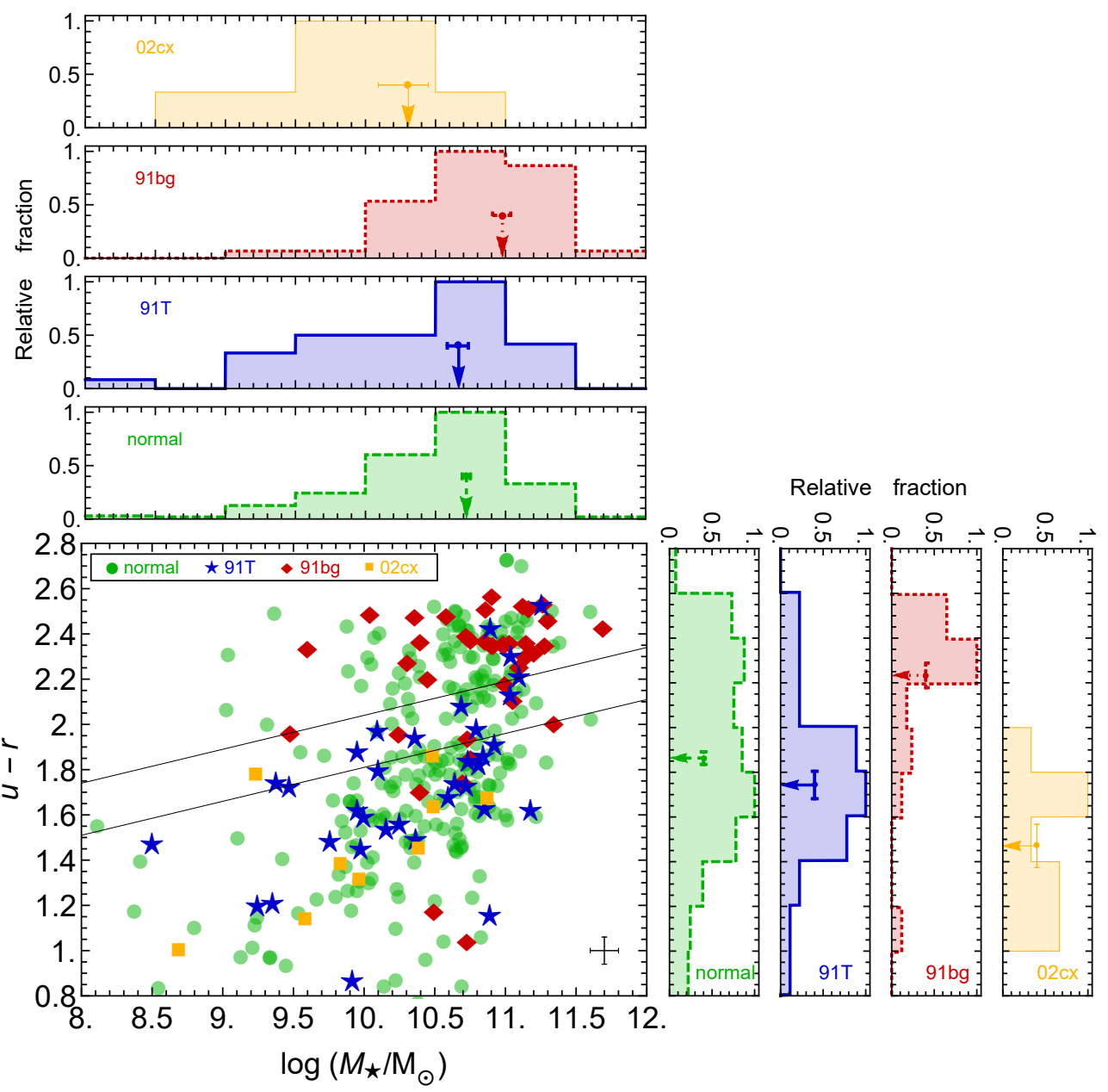

Figure 6. Colour-mass diagram for $326 \mathrm{SNe}$ Ia host galaxies with measured $u$ and $r$ magnitudes, displayed as scatter plots and distributions. In the bottom right corner of the lower left panel, the error bar represents the characteristic errors in our estimations of colours and masses of galaxies. The region between two solid lines marks the Green Valley (see text for details). For host galaxies of different SN subclasses, the right and upper panels show separately the histograms (distributions of relative fractions) of the colours and masses, respectively. The mean values (with standard errors) of the distributions are shown by arrows (with error bars).

Table 8. Comparison of the distributions of host galaxy $u-r$ colours and stellar masses among different SN Ia subclasses.

\begin{tabular}{|c|c|c|c|c|c|c|c|c|c|c|}
\hline \multicolumn{3}{|c|}{ Host subsample 1} & \multirow[t]{2}{*}{ vs } & \multicolumn{3}{|c|}{ Host subsample 2} & \multirow[t]{2}{*}{$P_{\mathrm{KS}}$} & \multirow[t]{2}{*}{$P_{\mathrm{AD}}$} & \multirow{2}{*}{$P_{\mathrm{KS}}^{\mathrm{MC}}$} & \multirow{2}{*}{$P_{\mathrm{AD}}^{\mathrm{MC}}$} \\
\hline SN subclass & $N_{\mathrm{SN}}$ & $\langle u-r\rangle$ & & SN subclass & $N_{\mathrm{SN}}$ & $\langle u-r\rangle$ & & & & \\
\hline normal & 244 & $1.86 \pm 0.03$ & vs & $91 \mathrm{~T}$ & 34 & $1.74 \pm 0.06$ & 0.064 & 0.092 & 0.060 & 0.101 \\
\hline normal & 244 & $1.86 \pm 0.03$ & vs & $91 \mathrm{bg}$ & 39 & $2.23 \pm 0.05$ & $<\mathbf{0 . 0 0 1}$ & $<\mathbf{0 . 0 0 1}$ & $<\mathbf{0 . 0 0 1}$ & $<0.001$ \\
\hline normal & 244 & $1.86 \pm 0.03$ & vs & $02 \mathrm{cx}$ & 9 & $1.47 \pm 0.10$ & 0.020 & 0.007 & 0.016 & 0.013 \\
\hline $91 \mathrm{~T}$ & 34 & $1.74 \pm 0.06$ & vs & $91 \mathrm{bg}$ & 39 & $2.23 \pm 0.05$ & $<\mathbf{0 . 0 0 1}$ & $<0.001$ & $<\mathbf{0 . 0 0 1}$ & $<\mathbf{0 . 0 0 1}$ \\
\hline $91 \mathrm{~T}$ & 34 & $1.74 \pm 0.06$ & vs & $02 \mathrm{cx}$ & 9 & $1.47 \pm 0.10$ & 0.140 & 0.041 & 0.117 & 0.059 \\
\hline $91 \mathrm{bg}$ & 39 & $2.23 \pm 0.05$ & vs & $02 \mathrm{cx}$ & 9 & $1.47 \pm 0.10$ & $<\mathbf{0 . 0 0 1}$ & $<0.001$ & $<\mathbf{0 . 0 0 1}$ & $<\mathbf{0 . 0 0 1}$ \\
\hline & & $\left\langle\log \left(M_{*} / \mathrm{M}_{\odot}\right)\right\rangle$ & & & & $\left.\log \left(M_{*} / \mathrm{M}_{\odot}\right)\right\rangle$ & & & & \\
\hline normal & 244 & $10.72_{-0.03}^{+0.03}$ & vs & $91 \mathrm{~T}$ & 34 & $10.66_{-0.08}^{+0.07}$ & 0.342 & 0.432 & 0.325 & 0.444 \\
\hline normal & 244 & $10.72_{-0.03}^{+0.03}$ & vs & $91 \mathrm{bg}$ & 39 & $10.98_{-0.07}^{+0.06}$ & $<0.001$ & $<0.001$ & $<0.001$ & $<0.001$ \\
\hline normal & 244 & $10.72_{-0.03}^{+0.03}$ & vs & $02 \mathrm{cx}$ & 9 & $10.30_{-0.21}^{+0.14}$ & $\mathbf{0 . 0 3 0}$ & 0.014 & 0.027 & 0.024 \\
\hline $91 \mathrm{~T}$ & 34 & $10.66_{-0.08}^{+0.07}$ & vs & $91 \mathrm{bg}$ & 39 & $10.98_{-0.07}^{+0.06}$ & 0.019 & 0.001 & 0.016 & 0.001 \\
\hline $91 \mathrm{~T}$ & 34 & $10.66_{-0.08}^{+0.07}$ & vs & $02 \mathrm{cx}$ & 9 & $10.30_{-0.21}^{+0.14}$ & 0.175 & 0.130 & 0.143 & 0.169 \\
\hline $91 \mathrm{bg}$ & 39 & $10.98_{-0.07}^{+0.06}$ & vs & $02 \mathrm{cx}$ & 9 & $10.30_{-0.21}^{+0.14}$ & 0.001 & $<0.001$ & $<\mathbf{0 . 0 0 1}$ & 0.001 \\
\hline
\end{tabular}

Notes. The explanations for $P$-values are the same as in Table 3. The statistically significant differences between the distributions are highlighted in bold. 

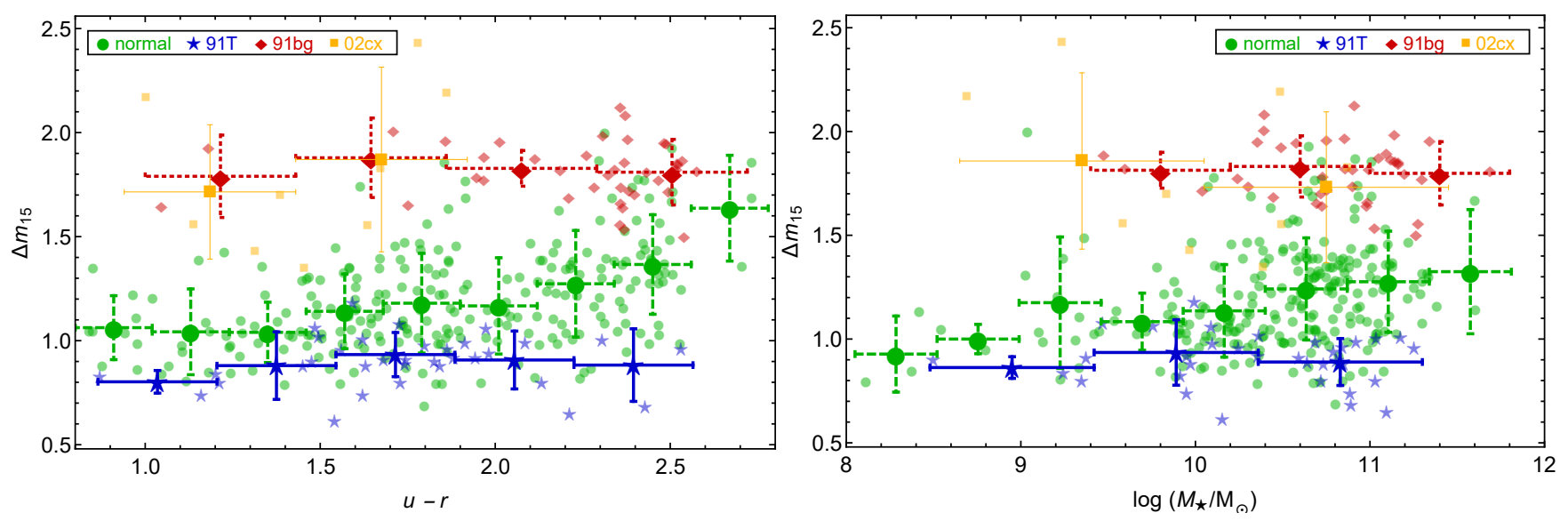

Figure 7. $B$-band $\Delta m_{15}$ values of the SN Ia subclasses versus host galaxy colour (left) and host galaxy stellar mass (right). Binned and averaged values of $\Delta m_{15}$ (bigger symbols) are superposed on the original distributions (smaller symbols). Horizontal bars show the bin sizes. Depending on the numbers of $\mathrm{SNe}$ Ia for certain subclasses, different binning sizes are selected to include sufficient numbers of the objects.

different SN subclasses. Host galaxies of 91bg-like SNe are clearly located in the Red Sequence, and most of them have $u-r$ colours $\gtrsim 2$ mag (i.e. above the Green Valley). In comparison with hosts of normal, 91T-, and 02cx-like SNe, the colour distribution of host galaxies of 91 bg-like $\mathrm{SNe}$ are significantly redder (see Table 8 ). Also, the bulk of hosts of $91 \mathrm{bg}$-like $\mathrm{SNe}$ are significantly massive $\left(\log \left(M_{*} / \mathrm{M}_{\odot}\right)>10.5\right)$. The distribution of host masses is significantly inconsistent with those of the other SN Ia subclasses (Table 8). At the same time, the colour (resp. mass) distributions are not statistically different between hosts of normal and 91T-like SNe, spanning almost the entire ranges of host colour (resp. mass). Finally, although the small number statistics, all the host galaxies of 02cx-like SNe are positioned in the Blue Cloud, mostly below the Green Valley in Fig. 6. Their colour (mass) distribution is significantly bluer (lower) in comparison with that of normal SNe Ia host galaxies, but closer to that of 91T-like SNe hosts (Table 8).

In Fig. 7, we show the distribution of the $B$-band $\Delta m_{15}$ values of the SN Ia subclasses as a function of $u-r$ colours and stellar masses of host galaxies. Interestingly, only normal SNe Ia show average systematic increase in $\Delta m_{15}$ values with an increase in colour (mass) values of hosts. The results of Spearman's rank test, shown in Table 9, confirm that only for normal SNe Ia we do see a significant correlation between LC decline rates and host galaxy colours (masses). We note that similar dependence of SNe Ia LC properties, such as decline rates, on the stellar masses of host galaxies have been shown in the literature using various SN Ia/host samples (e.g. Howell et al. 2009; Neill et al. 2009; Sullivan et al. 2010; González-Gaitán et al. 2011; Pan et al. 2014; Campbell et al. 2016; Uddin et al. 2017; Roman et al. 2018; Kelsey et al. 2020). However, instead of $\Delta m_{15}$, these studies used the SN Ia LC shape parameter $\Delta$ (adding and subtracting template LC shapes) or stretch $x_{1}$ (stretching or compressing the time axis of the LC by a single "stretch factor") obtained from two commonly used LC fitters in cosmology: MLCS2k2 (Jha, Riess \& Kirshner 2007) and SALT2 (Guy et al. 2007), respectively. These parameters $\left(\Delta\right.$ and $x_{1}$ ) show different correlations with the observed $\Delta m_{15}$ (e.g. Siebert et al. 2019). In addition, these studies did not perform a clear separation between normal and peculiar (91T-, 91bg-, and 02cx-like) SN Ia spectroscopic subclasses. The dependence of SNe Ia LC properties on the $u-r$ colours of host
Table 9. Results of Spearman's rank correlation test for the $B$-band $\Delta m_{15}$ values of the SN Ia subclasses versus $u-r$ colours and stellar masses of host galaxies.

\begin{tabular}{lrccccr}
\hline SN subclass & $N_{\mathrm{SN}}$ & Variable 1 & vs & Variable 2 & \multicolumn{1}{c}{$r_{\mathrm{s}}$} & \multicolumn{1}{c}{$P_{\mathrm{S}}$} \\
\hline normal & 244 & $\Delta m_{15}$ & vs & $u-r$ & 0.429 & $<\mathbf{0 . 0 0 1}$ \\
normal $^{a}$ & 149 & $\Delta m_{15}$ & vs & $u-r$ & 0.210 & $\mathbf{0 . 0 1 0}$ \\
normal $^{b}$ & 53 & $\Delta m_{15}$ & vs & $u-r$ & 0.285 & $\mathbf{0 . 0 3 8}$ \\
$91 \mathrm{~T}$ & 34 & $\Delta m_{15}$ & vs & $u-r$ & 0.143 & 0.421 \\
91bg & 39 & $\Delta m_{15}$ & vs & $u-r$ & -0.092 & 0.576 \\
02cx & 9 & $\Delta m_{15}$ & vs & $u-r$ & 0.417 & 0.264 \\
& & & & & & \\
normal $_{\text {normal }}{ }^{a}$ & 244 & $\Delta m_{15}$ & vs & $\log \left(M_{*} / \mathrm{M}_{\odot}\right)$ & 0.286 & $<\mathbf{0 . 0 0 1}$ \\
normal $^{b}$ & 149 & $\Delta m_{15}$ & vs & $\log \left(M_{*} / \mathrm{M}_{\odot}\right)$ & 0.159 & 0.052 \\
91T & 53 & $\Delta m_{15}$ & vs & $\log \left(M_{*} / \mathrm{M}_{\odot}\right)$ & 0.062 & 0.657 \\
91bg & 34 & $\Delta m_{15}$ & vs & $\log \left(M_{*} / \mathrm{M}_{\odot}\right)$ & -0.052 & 0.770 \\
02cx $^{\text {c }}$ & 39 & $\Delta m_{15}$ & vs & $\log \left(M_{*} / \mathrm{M}_{\odot}\right)$ & -0.091 & 0.582 \\
& 9 & $\Delta m_{15}$ & vs & $\log \left(M_{*} / \mathrm{M}_{\odot}\right)$ & -0.300 & 0.433 \\
\hline
\end{tabular}

Notes. The explanations for $r_{\mathrm{s}}$ and $P_{\mathrm{s}}$-values are the same as in Table 2 . ${ }^{a} \Delta m_{15} \lesssim 1.26 \mathrm{mag}$, similar to $91 \mathrm{~T}$-like SNe. ${ }^{b} \Delta m_{15} \gtrsim 1.38 \mathrm{mag}$, similar to 91 bg-like events. Statistically significant correlations between the variables are highlighted in bold.

galaxies was not directly studied in the literature, ${ }^{12}$ although it is expected, because similar dependencies on host morphology and other colours were known (e.g. Hamuy et al. 1996, 2000; Howell 2001; Gallagher et al. 2005; Jha et al. 2007; Ashall et al. 2016; Roman et al. 2018; Pruzhinskaya et al. 2020), again without clear separation between the spectroscopic subclasses of SNe Ia.

As in Subsection 3.2, for normal SNe Ia we check for the correlations between their $\Delta m_{15}$ and host galaxy properties in two separate $\Delta m_{15}$ ranges corresponding to the sizes of the $\Delta m_{15}$ ranges of 91T- and 91bg-like SNe. Table 9 shows that normal SNe Ia keep the $\Delta m_{15}$ - colour trend direction and significance in each of the narrower $\Delta m_{15}$ ranges, while the $\Delta m_{15}-\log \left(M_{*} / \mathrm{M}_{\odot}\right)$ trend becomes insignificant, hinting that the $\Delta m_{15}$ ranges might play a role in the absence of the $\Delta m_{15}-\log \left(M_{*} / \mathrm{M}_{\odot}\right)$ trend for peculiar SNe Ia.

12 But see Kelsey et al. (2020), for indirectly obtained $U-R$ colours of SNe Ia host galaxies. 
Table 10. Comparison of the $B$-band $\Delta m_{15}$ distributions of only normal SNe Ia among different subsamples of host parameters.

\begin{tabular}{|c|c|c|c|c|c|c|c|c|c|c|c|c|}
\hline \multicolumn{4}{|c|}{ Subsample 1} & \multirow[t]{2}{*}{ vs } & \multicolumn{4}{|c|}{ Subsample 2} & \multirow[t]{2}{*}{$P_{\mathrm{KS}}$} & \multirow[t]{2}{*}{$P_{\mathrm{AD}}$} & \multirow[t]{2}{*}{$P_{\mathrm{KS}}^{\mathrm{MC}}$} & \multirow[t]{2}{*}{$P_{\mathrm{AD}}^{\mathrm{MC}}$} \\
\hline Host para & neter & $N_{\mathrm{SN}}$ & $\left\langle\Delta m_{15}\right\rangle$ & & Host paral & neter & $N_{\mathrm{SN}}$ & $\left\langle\Delta m_{15}\right\rangle$ & & & & \\
\hline$u-r$ & $>2 \mathrm{mag}$ & 100 & $1.30 \pm 0.02$ & vs & $u-r$ & $\leqslant 2 \mathrm{mag}$ & 144 & $1.13 \pm 0.02$ & $<\mathbf{0 . 0 0 1}$ & $<0.001$ & $<\mathbf{0 . 0 0 1}$ & $<\mathbf{0 . 0 0 1}$ \\
\hline $\log \left(M_{*} / \mathrm{M}_{\odot}\right)$ & $>10.5$ & 139 & $1.26 \pm 0.02$ & vs & $\log \left(M_{*} / \mathrm{M}_{\odot}\right)$ & $\leqslant 10.5$ & 105 & $1.13 \pm 0.02$ & $<\mathbf{0 . 0 0 1}$ & $<0.001$ & $<\mathbf{0 . 0 0 1}$ & $<\mathbf{0 . 0 0 1}$ \\
\hline \multicolumn{2}{|c|}{ above Green Valley } & 81 & $1.33 \pm 0.03$ & vs & \multicolumn{2}{|c|}{ below/in Green Valley } & 163 & $1.14 \pm 0.02$ & $<0.001$ & $<0.001$ & $<\mathbf{0 . 0 0 1}$ & $<\mathbf{0 . 0 0 1}$ \\
\hline \multicolumn{2}{|c|}{ above Green Valley } & 81 & $1.33 \pm 0.03$ & vs & \multicolumn{2}{|c|}{ below Green Valley } & 133 & $1.13 \pm 0.02$ & $<0.001$ & $<0.001$ & $<0.001$ & $<0.001$ \\
\hline
\end{tabular}

Notes. The explanations for $P$-values are the same as in Table 3. A 0.15 mag and 0.25 dex variations in colour and mass demarcations, respectively, do not change the statistical behaviour of the tests. The statistically significant differences between the distributions are highlighted in bold.

Without mixing the SN Ia subclasses, we compare in Table 10 the distributions of LC decline rates of normal SNe Ia between two distinct populations of host galaxies in the colour-mass diagram. The separations between two populations of galaxies are done according to $u-r$ colour (e.g. Schawinski et al. 2014), stellar mass (e.g. Uddin et al. 2017), or according to locations of galaxies above and below the Green Valley. Indeed, the Green Valley separates best the two populations of passively evolving quenched (older) galaxies and of star-forming (younger) hosts. Table 10 shows that the distributions of $\Delta m_{15}$ values of normal SNe Ia are significantly different between all the mentioned host subsamples. On average, those normal SNe Ia that are in galaxies above the Green Valley have faster declining LCs compared to those in galaxies below the Green Valley. This result suggests that the correlation seen in Fig. 7 and Table 9 is due to a superposition of faster and slower declining normal SNe Ia from old and young stellar populations of host galaxies, respectively dominating in the Red Sequence and Blue Cloud of the colour-mass diagram (see Fig. 6). In addition to the findings in Subsection 3.2, the colour-mass study of SN Ia hosts also suggest that there could be at least two distinct populations of normal SNe Ia: the halo/bulge and old disc components of galaxies most likely host faster declining normal SNe Ia, while star-forming component of galaxies hosts their slower declining counterparts.

Taken separately, the LC decline rates of 91bg-like SNe (subluminous SNe Ia) and 91T-like events (overluminous SNe Ia) do not show dependencies on host galaxy colour (Fig. 7 and Table 9). At the same time, the distribution of hosts on the colour-mass diagram confirms the known tendency for 91bg-like SNe to occur in globally red/old galaxies while 91T-like events prefer blue/younger hosts. Therefore, the results in Figs. 5, 6, and Table 8 reinforce those obtained in Subsection 3.2 that among the considered SN Ia subclasses, 91bglike SNe come only from old stellar population of hosts (halo/bulge and old disc components), while 91T-like events originate only from young population of galaxies (star-forming component). Probably, the decline rates of $02 \mathrm{cx}$-like $\mathrm{SNe}$ also do not show dependencies on host galaxy properties (Table 9), although we note that statistics of these events is based on small numbers. Complementing the results of Subsection 3.2, the positions of 02cx-like SNe hosts in the colour-mass diagram suggest that these events may all originate from the young stellar component, although they diverge from 91Tlike events in their significantly higher LC decline rate.

Since the $g$ - and $i$-bands photometry is available for all $407 \mathrm{SNe} \mathrm{Ia}$ host galaxies, we also check the stellar mass-based results in Tables 8-10 using the entire SN Ia subsamples. As a result, all behaviours of the dependencies $\left(\Delta m_{15}\right.$ vs $\left.\log \left(M_{*} / \mathrm{M}_{\odot}\right)\right)$ and statistical significances of the tests remain almost unchanged.

Interestingly, some studies suggested that the correlations between the optically-based LC properties and host galaxy mass might be due to differences in dust extinction in galaxies with different masses (e.g. Burns et al. 2018; Brout \& Scolnic 2020). However,

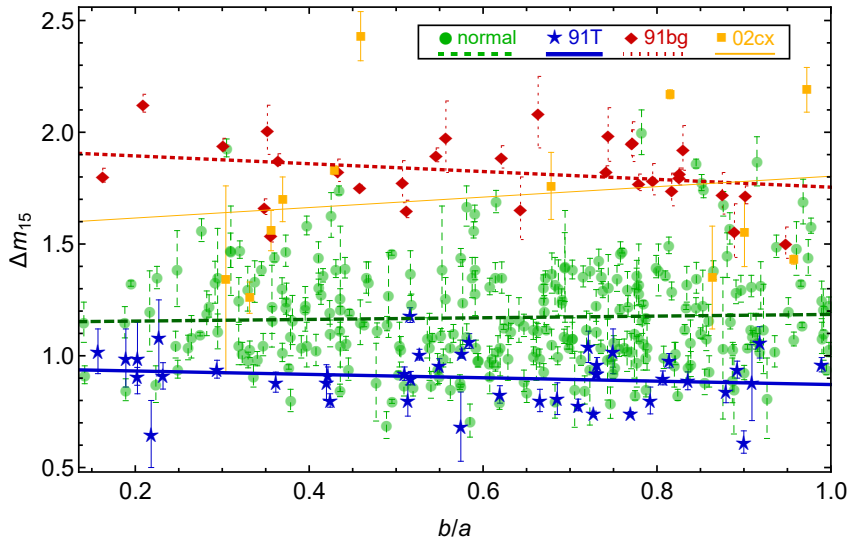

Figure 8. Distributions of the $B$-band $\Delta m_{15}$ values of different subclasses of $360 \mathrm{SNe}$ Ia as a function of $b / a$ of S0-Sm host galaxies (a measure of galaxy inclination), and their best-fitting lines.

most recently Uddin et al. (2020) observed near-constant correlations between SN Ia LC properties and host galaxy mass across nearinfrared, which are less sensitive to dust, and optical bands. They suggested that dust extinction might not play a significant role in the observed correlation (see also Ponder et al. 2020). In this respect, the combination of the lack/non-significance of $\Delta m_{15}$ - host stellar mass (morphology, colour) correlations for peculiar SNe Ia and the observed significant correlation for normal SNe Ia (Figs. 4, 7 and Table 9) also suggest that dust may not be the dominant process in the correlations of LCs and host properties for normal SNe Ia. If the impact of dust were strong, then one would expect that, contrary to what we found in Fig. 4, the $B$-band $\Delta m_{15}$ of 91T-like SNe would be correlated with host galaxy properties, because the bulk of these events are discovered in star-forming galaxies with different masses and dust properties.

Indeed, one could also expect that SNe Ia in strongly inclined hosts are more extinguished and will show smaller $\Delta m_{15}$ (see Subsection 2.1). Fig. 8 shows the distribution of $\Delta m_{15}$ versus $b / a$ of S0-Sm host galaxies, ${ }^{13}$ which is a measure of galaxy inclination. However, the Spearman's rank test shows that these variables are not correlated: the $P_{\mathrm{s}}$-values are $0.745,0.419,0.123$, and 0.275 for normal, 91T-, 91bg-, and 02cx-like $\mathrm{SNe}$, respectively (see also Uddin et al. 2017). Therefore, we believe that the observed normal SN Iahost relations are dominated by the diversity of SNe Ia progenitors.

13 The elongations $(b / a)$ of host galaxies are obtained from the fitted $25 \mathrm{mag} \operatorname{arcsec}^{-2}$ elliptical apertures in the $g$-band (see Subsection 2.2). 


\subsection{Luminosity-weighted ages of SNe Ia host galaxies}

In order to move from the qualitative description of the progenitor population ages of the SN Ia subclasses to the quantitative ones, we determine the luminosity-weighted ages of their host galaxies. ${ }^{14}$ To conform to values used in the previous paper of our series (Barkhudaryan et al. 2019), we use the fixed redshifts of the galaxies to fit the PÉGASE. 2 templates library (Fioc \& Rocca-Volmerange 1997, 1999), comprised of various morphological/spectral types, to the measured ugriz photometry of SN hosts. Five photometric values of a host galaxy (in some cases only four griz band values are available, see Subsection 2.2) and its fixed redshift are used to select the best locations of the values on the spectral energy distribution (SED) templates. Accordingly, the best matched SED model and corresponding luminosity-weighted age can be used from the collection of all possible synthetic templates for different galaxy ages (up to $19 \mathrm{Gyr}$ ). The luminosity-weighted stellar population ages are very sensitive to recent star formation, i.e. bright young stellar populations get more weight in the estimated ages. An example of SN Ia host galaxy photometry with its best template, and more information on the SED fitting method can be found in Barkhudaryan et al. (2019).

In the upper panel of Fig. 9, we present the cumulative distributions of luminosity-weighted ages of normal and peculiar (91T-, 91bg-, and 02cx-like) SNe Ia host galaxies. Note that only about 10 per cent of the host galaxies in our sample have very late-type morphologies (Scd-Sm, see Table 4) that generally exhibit stellar populations younger than a few Gyr (e.g. González Delgado et al. 2015). Therefore, we have a deficit of hosts with ages less than a few Gyr in our age calculations. The two-sample KS and AD tests in Table 11 show that the age distribution of normal SNe Ia hosts is consistent with that of 91T-like SNe hosts, but significantly inconsistent with those of $91 \mathrm{bg}$ - and $02 \mathrm{cx}$-like SNe hosts. In comparison with hosts of normal and 91T-like SNe, the ages of 91bg-like SNe hosts are older on average, while the ages of hosts of $02 \mathrm{cx}$-like events are younger. These results are expected in light of those in Fig. 6 and Table 8, when considering the $u-r$ colour as a proxy for recent star formation or stellar population ages of galaxies.

In the bottom panel of Fig. 9, we show the distribution of the $B$-band $\Delta m_{15}$ values of the $\mathrm{SN}$ Ia subclasses as a function of luminosity-weighted ages of host galaxies. In analogy with the results in Table 9, the Spearman's rank test, presented in Table 12, shows that the significant correlation exists only between normal SNe Ia LC decline rates and stellar population ages of their host galaxies. These $\mathrm{SNe}$ Ia show an average increase in $\Delta m_{15}$ values with an increase in host's age. Using the SN Ia LC stretch instead of $\Delta m_{15}$, similar correlations between the SN LC and host parameters have been shown many times in the literature (e.g. Neill et al. 2009; Gupta et al. 2011; Johansson et al. 2013; Pan et al. 2014; Campbell et al. 2016; Kang et al. 2020). The samples of these studies mostly include normal SNe Ia events, thus not studying separately the correlations for peculiar (91T-, 91bg-, and 02cx-like) SN Ia subclasses. While, importantly we show that these peculiar SNe Ia subclasses originate from host galaxies diverging in age (Table 11), however taken separately their LC decline rates do not show dependencies on host galaxy age, contrary to normal SNe Ia events (Table 12). These results are in good support of those obtained in Subsections 3.23.3 that $91 \mathrm{bg}$-like $\mathrm{SNe}$ come only from old stellar population of hosts, while 91T-like events originate only from young population

14 The luminosity-weighted ages of host galaxies are available in the online version of this article (Supporting Information).
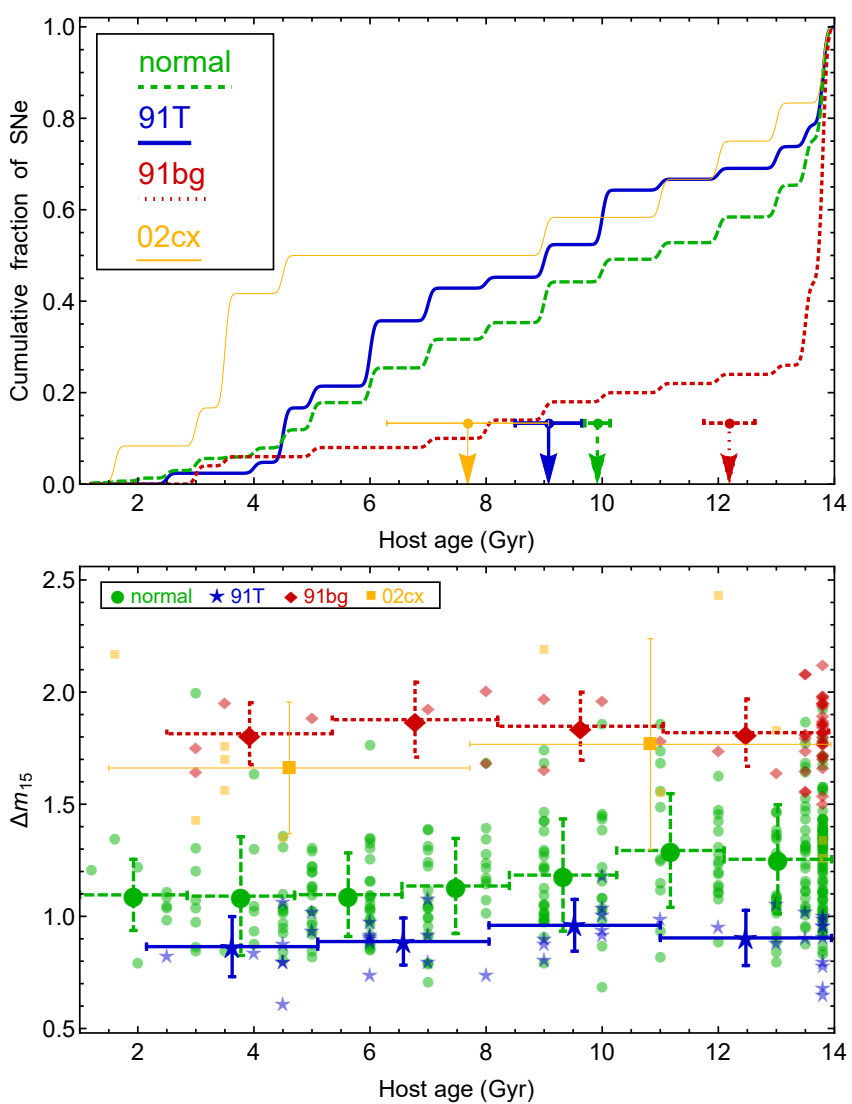

Figure 9. Upper panel: cumulative distributions of SNe Ia host galaxy luminosity-weighted ages. The mean ages (with standard errors) of the host subsamples are shown by arrows (with error bars). Bottom panel: $B$-band $\Delta m_{15}$ values of the SN Ia subclasses versus luminosity-weighted ages of host galaxies. Binned and averaged values of $\Delta m_{15}$ (bigger symbols) are superposed on the original distributions (smaller symbols). Horizontal bars show the bin sizes. Depending on the numbers of SNe Ia for certain subclasses, different binning sizes are selected to include sufficient numbers of the objects.

of galaxies, thus likely belonging to two unique classes of progenitors.

In Table 12, for normal SNe Ia we check for the $\Delta m_{15}$ age correlation in the two separate and narrower $\Delta m_{15}$ ranges. In the slower declining range, normal SNe Ia keep the $\Delta m_{15}$ - age trend direction and significance, while in the faster declining range, the trend becomes insignificant, hinting that the $\Delta m_{15}$ ranges and/or small number statistics might play some role in the absence of the $\Delta m_{15}$ age trend for 91T- and 91bg-like SNe.

Interestingly, to study the connection between SNe Ia and their host characteristics, Rose, Garnavich \& Berg (2019) estimated the hosts' global stellar population ages as well as the local environment ages around the sites of photometrically and spectroscopically classified SDSS II SNe Ia (103 events, $z<0.2$ ). The authors noted that the significant correlation between SN Ia LC properties/luminosity and host stellar population age might be an effect of age step at $\sim 8$ Gyr (corresponding to the jump in the average LC properties). Rose et al. also noted that both the local environment age and global one show the equivalent correlations with the $\mathrm{SNe}$ properties, however, as expected, the age of the local stellar population is younger than that of the global one for SN Ia in star-forming environments. With that in mind, we split the sample of normal SNe Ia into 
Table 11. Comparison of the distributions of host galaxy luminosity-weighted ages among different SN Ia subclasses.

\begin{tabular}{|c|c|c|c|c|c|c|c|c|c|c|}
\hline \multicolumn{3}{|c|}{ Host subsample 1} & \multirow[t]{2}{*}{ vs } & \multicolumn{3}{|c|}{ Host subsample 2} & \multirow[t]{2}{*}{$P_{\mathrm{KS}}$} & \multirow[t]{2}{*}{$P_{\mathrm{AD}}$} & \multirow{2}{*}{$P_{\mathrm{KS}}^{\mathrm{MC}}$} & \multirow{2}{*}{$P_{\mathrm{AD}}^{\mathrm{MC}}$} \\
\hline SN subclass & $N_{\mathrm{SN}}$ & $\langle$ host age $\rangle$ & & SN subclass & $N_{\mathrm{SN}}$ & $\langle$ host age $\rangle$ & & & & \\
\hline normal & 303 & $9.9 \pm 0.2$ & vs & $91 \mathrm{~T}$ & 42 & $9.1 \pm 0.6$ & 0.211 & 0.310 & 0.211 & 0.325 \\
\hline normal & 303 & $9.9 \pm 0.2$ & vs & $91 \mathrm{bg}$ & 50 & $12.2 \pm 0.4$ & $<0.001$ & $<\mathbf{0 . 0 0 1}$ & $<\mathbf{0 . 0 0 1}$ & $<0.001$ \\
\hline normal & 303 & $9.9 \pm 0.2$ & vs & $02 \mathrm{cx}$ & 12 & $7.7 \pm 1.4$ & 0.029 & 0.016 & 0.030 & 0.030 \\
\hline $91 \mathrm{~T}$ & 42 & $9.1 \pm 0.6$ & vs & $91 \mathrm{bg}$ & 50 & $12.2 \pm 0.4$ & $<0.001$ & $<0.001$ & $<\mathbf{0 . 0 0 1}$ & $<0.001$ \\
\hline $91 \mathrm{~T}$ & 42 & $9.1 \pm 0.6$ & vs & $02 \mathrm{cx}$ & 12 & $7.7 \pm 1.4$ & 0.052 & 0.038 & 0.051 & 0.049 \\
\hline $91 \mathrm{bg}$ & 50 & $12.2 \pm 0.4$ & vs & $02 \mathrm{cx}$ & 12 & $7.7 \pm 1.4$ & 0.001 & $<\mathbf{0 . 0 0 1}$ & 0.001 & 0.002 \\
\hline
\end{tabular}

Notes. The explanations for $P$-values are the same as in Table 3 . The statistically significant differences between the distributions are highlighted in bold.

Table 12. Results of Spearman's rank correlation test for the $B$-band $\Delta m_{15}$ values of the SN Ia subclasses versus luminosity-weighted ages of host galaxies.

\begin{tabular}{lrccccc}
\hline SN subclass & $N_{\text {SN }}$ & Variable 1 & vs & Variable 2 & \multicolumn{1}{c}{$r_{\mathrm{S}}$} & \multicolumn{1}{c}{$P_{\mathrm{S}}$} \\
\hline normal & 303 & $\Delta m_{15}$ & vs & host age & 0.314 & $<\mathbf{0 . 0 0 1}$ \\
normal $^{a}$ & 189 & $\Delta m_{15}$ & vs & host age & 0.161 & $\mathbf{0 . 0 2 7}$ \\
normal $^{b}$ & 64 & $\Delta m_{15}$ & vs & host age & -0.059 & 0.644 \\
$91 \mathrm{~T}$ & 42 & $\Delta m_{15}$ & vs & host age & 0.154 & 0.330 \\
$91 \mathrm{bg}$ & 50 & $\Delta m_{15}$ & vs & host age & 0.082 & 0.569 \\
$02 \mathrm{cx}$ & 12 & $\Delta m_{15}$ & vs & host age & -0.264 & 0.406
\end{tabular}

Notes. The explanations for $r_{\mathrm{s}}$ and $P_{\mathrm{S}}$-values are the same as in Table 2 . ${ }^{a} \Delta m_{15} \lesssim 1.26 \mathrm{mag}$, similar to $91 \mathrm{~T}$-like SNe. ${ }^{b} \Delta m_{15} \gtrsim 1.38 \mathrm{mag}$, similar to $91 \mathrm{bg}$-like events. Statistically significant correlations between the variables are highlighted in bold.

two subsamples according to the host age step at $8 \mathrm{Gyr}$, and compare the $\Delta m_{15}$ distributions between the subsamples $\left(N_{\mathrm{SN}}=107\right.$, $\left\langle\Delta m_{15}\right\rangle=1.10 \pm 0.02$ for host age $\leqslant 8 \mathrm{Gyr}$ versus $N_{\mathrm{SN}}=196$, $\left\langle\Delta m_{15}\right\rangle=1.24 \pm 0.02$ for host age $>8 \mathrm{Gyr}$ ). The two-sample $\mathrm{KS}$ and $\mathrm{AD}$ tests show that the $\Delta m_{15}$ distributions are significantly inconsistent between each other $\left(P_{\mathrm{KS}}^{\mathrm{MC}}<0.001, P_{\mathrm{AD}}^{\mathrm{MC}}<0.001\right)$. As expected from Fig. 9 and Table 12, galaxies with ages $\leqslant 8 \mathrm{Gyr}$ host on average slower declining/brighter SNe Ia (spectroscopically normal) in comparison with older hosts (see also Shen, Toonen \& Graur 2017). Note that a 3 Gyr variation in the host age step does not change the statistical behaviour of the tests. Thus, the significant correlation seen in Table 12 is due to a superposition of faster and slower declining normal $\mathrm{SNe}$ Ia from older and younger stellar components of hosts, respectively (see also Rigault et al. 2013, as measured by the environmental $\mathrm{H} \alpha$ emission at the positions of $\mathrm{SNe}$ Ia).

This result can be explained considering the correlation between ${ }^{56} \mathrm{Ni}$ mass synthesized in SN Ia and host galaxy age (e.g. Howell et al. 2009). In this respect, Neill et al. (2009) suggested that the details of the ${ }^{56} \mathrm{Ni}$ mass - age correlations in their study and in Howell et al. (2009) imply an age step/threshold of about several Gyr for $\mathrm{SN}$ Ia hosts, above which galaxies are less likely to produce SNe Ia with ${ }^{56} \mathrm{Ni}$ masses $\gtrsim 0.5 M_{\odot}$, i.e. slower declining/brighter events.

It is important also to mention that the $\Delta m_{15}$ - morphology, $\Delta m_{15}-$ colour, and $\Delta m_{15}-\log \left(M_{*} / \mathrm{M}_{\odot}\right)$ correlations, we showed in previous subsections for spectroscopically normal SN Ia, and the $\Delta m_{15}$ age correlation agree with one another in the sense that host galaxy morphology, colour, mass, and age are correlated, with older galaxies mainly being earlier type, redder, and more massive (e.g. González Delgado et al. 2015). Therefore, the previously reported correlations between LC properties of SNe Ia and physical parameters of their host galaxies are most likely originated from the dif- ferences in host stellar population ages (see also earlier discussions in Gupta et al. 2011; Rigault et al. 2013; Childress et al. 2014; Rose et al. 2019; Kang et al. 2020).

\subsection{Constraints on delay time distributions of SNe Ia}

Current theoretical models of spectroscopically normal SNe Ia progenitors predict that most likely their DTD peaks below/close to $1 \mathrm{Gyr}$, corresponding to the young/prompt component, then declines fast by over two orders of magnitude at several Gyr (e.g. Yungelson \& Livio 2000; Greggio 2005; Childress et al. 2014). At redshifts near zero (as in our sample), the SN Ia rate in evolved galaxies, from a large volume, results in a bimodal shape of the distribution of SN Ia progenitor ages, with assumption that galaxies have nearly constant SFR (e.g. Childress et al. 2014). In such a bimodal age distribution, the second peak includes old/delayed events at current epoch, which are naturally absent at high redshifts. Young/prompt SNe Ia originate in actively star-forming galaxies, predominantly from progenitors whose ages correspond to the first peak of the SN Ia progenitor age distribution. While old/delayed SNe Ia originate in galaxies that already have an old stellar component or they are entirely old whose star formation ceased in the past. In such old stellar systems, the DTD is lacking young/prompt events, meaning that delayed SNe Ia occur from different age group of progenitors (e.g. Childress et al. 2014; Maoz et al. 2014). In this respect, the significant correlation between the LC decline rates and the global host galaxy ages of normal SNe Ia (see Subsection 3.4) is likely caused by the bimodal behaviour of the age distribution of normal SN Ia progenitors, which includes both the prompt events from young progenitors (slower declining $\mathrm{SNe}$ Ia) and delayed $\mathrm{SNe}$ Ia from old systems (faster declining events). On the other hand, the absence (non-significance) of the mentioned correlation for peculiar (91T-, 91bg-, and 02cxlike) SNe Ia and the observed differences in the properties of their host galaxies (see Subsections 3.2 and 3.3) can be considered as evidences for distinct (single-mode) behaviours of their progenitor age distribution, thus distinct progenitor channels.

Our results on peculiar 91bg-like events agree with those of González-Gaitán et al. (2011), who found that subluminous/91bglike $\mathrm{SNe}(18$ events with $z \leqslant 0.6$ ) in their photometrically identified SNe sample are mainly found in early-type hosts with almost no star formation, and argued that these SNe come from a delayed progenitor component with a Gaussian DTD centered around $6 \mathrm{Gyr}$, but with large uncertainty up to $\sim 11$ Gyr. Recently, Panther et al. (2019) studied integral field observations of the hosts' explosion sites of 11 spectroscopically identified $91 \mathrm{bg}$-like events $(z \leqslant 0.04)$ and found that the majority of the stellar populations in the vicinity of these SNe locations are dominated by old stars with a lack of recent star formation. The authors concluded that the DTD of 91bg-like SNe 
is likely weighted toward long delay times, larger than $\sim 6 \mathrm{Gyr}$ (see also Crocker et al. 2017). Most recently, in Barkhudaryan et al. (2019) we found that in elliptical host galaxies of SNe Ia, the age distribution of 91bg-like SNe hosts ( $28 \mathrm{Gyr}$ ) does not extend down to the stellar ages that produce significant $u$-band fluxes of their early-type hosts, contrary to the hosts of normal SNe Ia. Therefore, younger stars in elliptical galaxies do not produce 91bg-like $\mathrm{SNe}$.

Our study includes the largest sample of 50 subluminous $91 \mathrm{bg}$ like $\mathrm{SNe}$ in a much wider morphological range of host galaxies than previous studies, and shows even more clearly that the progenitor population age of these events is strongly weighted toward very old ages (oldest among the other SN Ia subclasses, see Table 11 and Fig. 9). More than 85 per cent of 91bg-like SNe hosts are older than 8 Gyr. As already mentioned, such delay times are much longer than the delays of normal Type Ia $\mathrm{SNe}$ from star-forming environments, whose DTD peaks between several hundred Myr and $\sim 1$ Gyr (a sharp initial peak) with a low tail at higher delay times (e.g. Childress et al. 2014). Therefore, most likely $91 \mathrm{bg}$-like events originate exclusively from old stellar population of host galaxies, and thus these peculiar SNe Ia have no prompt component.

As in Barkhudaryan et al. (2019), we favour the DD channel for the progenitors of these peculiar events that belong to the old stellar components of galaxies (see also Ruiter et al. 2009; Gilfanov \& Bogdán 2010; Lipunov et al. 2011): in particular the progenitor models such as He-ignited violent mergers (CO WD primary with He WD companion) might be appropriate for $91 \mathrm{bg}$-like events (e.g. Pakmor et al. 2013; Crocker et al. 2017). These models predict very long delay times for subluminous 91bg-like SNe ( On the other hand, the DD channel with CO WD primary and CO WD companion (e.g. Pakmor et al. 2013) can provide an appropriate DTD for normal SNe Ia with an initial peak below/close to $1 \mathrm{Gyr}$ and a tail up to about $10 \mathrm{Gyr}$, while most SD models predict few or no SNe Ia at long delays (e.g. Childress et al. 2014; Maoz et al. 2014). In addition, Perets et al. (2019) showed that mergers of CO WD with hybrid He-CO WD could also give rise to normal Type Ia SNe whose synthetic LCs and spectra are consistent with those of observed SNe Ia (see also Zenati, Toonen \& Perets 2019, for the properties of hybrid He-CO WDs). These authors noted that together with the contribution from mergers of massive double CO WDs that give rise to more luminous $\mathrm{SNe}$ Ia, their models can potentially reproduce the full range of normal SNe Ia, their DTD and rate.

Relatively younger global host ages of overluminous 91T-like $\mathrm{SNe}$ (Fig. 9) can be explained in the context of shorter DTD in comparison with that of 91bg-like events. In addition, 91T-like SNe strongly prefer blue (Fig. 6) and late-type hosts (Fig. 4), and no such an event is observed in old elliptical galaxies (Table 4), thus supporting shorter, i.e. relatively prompt DTD for these SNe. In this sense, the DTD of the SD channel is different from that of DD one and can be appropriate for 91T-like SNe (e.g. Fisher \& Jumper 2015). The DTD of SD channel has a sharp cutoff at 2-3 Gyr, because in its original formulation a donor/companion star in an SD system is a main sequence or red giant star with only a narrow range of masses that could provide mass accretion at the sufficient rate resulting in a stable burning and an explosion of WD (e.g. Han \& Podsiadlowski 2004). Also, note that massive CO WD primaries in DD models are naturally explode in more luminous SNe Ia (e.g. overluminous/91Tlike events). Such massive WDs, which originated from more massive intermediate-mass stars, in DD systems have delay times $\lesssim$ several hundred Myr (e.g. the violent WDs merger scenario; Ruiter et al. 2013), and is therefore expected in late-type star-forming galaxies.

These DTDs may seem too young in comparison with the global age distribution of hosts (see Fig. 9 and Table 11), however, as men- tioned in Subsection 3.4 for normal SN Ia in star-forming environments, the age of the local stellar population at the SN position is younger (on average) than the global age of its host galaxies (e.g. Rose et al. 2019). Therefore, in an ideal case, the stellar population age obtained from the location of the $\mathrm{SN}$ in the host galaxy would be preferable to the global host age that we estimate in the current study (see also discussions in Gupta et al. 2011; Fisher \& Jumper 2015). Consequently, additional local age-estimations are needed for 91T-like SNe to better test the DTDs of the SD and DD progenitors models.

Despite the small number statistics of $02 \mathrm{cx}$-like $\mathrm{SNe}$, on average their host galaxies have the youngest global ages (Fig. 9), latest morphology (Fig. 4), bluest colour and lowest masses (Fig. 6) among the other SN Ia subclasses, again strongly pointing to a shorter DTD for the events. In our sample the host galaxy properties of $02 \mathrm{cx}$-like $\mathrm{SNe}$ are close to those of star-forming hosts of 91T-like SNe (see also Foley et al. 2009; Perets et al. 2010), however, their LC decline rates are significantly different from those of 91T-like events (Fig. 3 and Table 5) and fall off the SN Ia LC width-luminosity relation (see e.g. Taubenberger 2017). The properties of 02cx-like SNe and evidence of their short DTD suggest a binary system where 02cx-like $\mathrm{SN}$ arises from massive CO WD that quickly accretes helium from an He-star donor (e.g. Postnov \& Yungelson 2014). Then accretiontriggered explosion of a Chandrasekhar mass WD does not necessarily fully disrupt the star. Among a variety of proposed scenarios, this is now the leading model (see review by Jha 2017, and references therein). This channel might be the dominant one for delays of up to a few hundred Myr, above which hydrogen-accreting SD and DD systems dominate (e.g. Claeys et al. 2014). Again, the stellar population ages obtained at the locations of 02cx-like SNe in the host galaxies would be preferable to the global host ages to better test the DTD of the leading model.

\section{CONCLUSIONS}

In the seventh paper of a series, using a well-defined sample of Type Ia SNe and their host galaxies, we comparatively analyse the relations between the LC decline rates and the global properties of hosts of various SN Ia subclasses to better understand the diverse nature of SNe Ia progenitors. The spectroscopic subclasses of SNe Ia (normal, 91T-, 91bg-, and 02cx-like) and their B-band LC decline rates $\left(\Delta m_{15}\right)$ are carefully compiled from the available literature, while the data of the SNe Ia host galaxies is a homogeneous set of consistent measurements performed by the authors of this study. Our sample consists of 394 relatively nearby ( $\leqslant 150 \mathrm{Mpc}$, the mean distance is $72 \mathrm{Mpc}$ ) E-Sm galaxies, which host $407 \mathrm{SNe}$ Ia in total.

There is no strong redshift bias within our sample, which could drive the observed relations between global properties of hosts and extinction-independent LC decline rates of the SN Ia subclasses. In addition, the representation of $\mathrm{SN}$ subclasses in our compilation and in nearly complete volume-limited sample of the LOSS are not different statistically. However, due to the small number statistics of 02cx-like $\mathrm{SNe}$, the results on these peculiar events and their hosts should be considered with caution.

All the obtained results and their interpretations are summarized below.

(i) In general, the $B$-band $\Delta m_{15}$ distribution of $\mathrm{SNe}$ Ia seems to be bimodal (Fig. 3), with the second (weaker) mode mostly distributed within $\sim 1.5$ to $2.1 \mathrm{mag}$. This faster declining range is generally occupied by $91 \mathrm{bg}$-like (subluminous) events, while the $\Delta m_{15}$ of 91T-like (overluminous) SNe are distributed only within the first 
mode at slower declining range $\left(\Delta m_{15} \lesssim 1.1 \mathrm{mag}\right)$. The decline rates of $02 \mathrm{cx}$-like $\mathrm{SNe}$ are spread on the faster side of the $\Delta m_{15}$ distribution of normal SNe Ia. Statistically, all these distributions are significantly different from one another (Table 5).

(ii) The host galaxies of normal, 91T-, and 91bg-like SNe Ia have morphological type distributions that are significantly inconsistent between one another (Fig. 4 and Table 6). Hosts of 91bg-like SNe have, on average, earlier morphological types $(\langle t\rangle \approx 0)$ with a large number of the events discovered in E-S0 galaxies. While hosts of 91T-like SNe have on average later morphological types $(\langle t\rangle \approx 4)$ with a single 91T-like event in the E-S0 bin (Table 4). The same distribution of hosts of normal SNe Ia occupies an intermediate position between the host morphologies of 91T- and 91bg-like events. The morphological distribution of $02 \mathrm{cx}$-like $\mathrm{SNe}$ hosts is similar to that of 91T-like SNe hosts.

(iii) As for galaxies in general, the distribution of $\mathrm{SNe}$ Ia hosts in the $u-r$ colour-mass diagram is bimodal (Fig. 5). The hosts of 91bg-like $\mathrm{SNe}$ are located in the Red Sequence of the diagram, most of them have $u-r$ colours $\gtrsim 2$ mag (i.e. above the Green Valley). In comparison with hosts of normal, 91T-, and 02cx-like SNe, the colour distribution of hosts of $91 \mathrm{bg}$-like SNe are significantly redder (Table 8). Importantly, the bulk of hosts of $91 \mathrm{bg}$-like SNe are significantly massive $\left(\log \left(M_{*} / \mathrm{M}_{\odot}\right)>10.5\right)$ and old (more than 10 Gyr). The hosts' mass (age) distribution is significantly inconsistent with those of the other SN Ia subclasses (Tables 8 and 11). At the same time, the colour (mass, age) distributions are not statistically different between hosts of normal and 91T-like SNe. Finally, all the host galaxies of $02 \mathrm{cx}$-like $\mathrm{SNe}$ are positioned in the Blue Cloud of the colour-mass diagram, mostly below the Green Valley (Fig. 6). Their colour (mass, age) distribution is significantly bluer (lower, younger) in comparison with that of normal $\mathrm{SNe}$ Ia hosts, but closer to that of 91T-like SNe hosts.

(iv) As previously shown with smaller nearby SN Ia samples, there is a significant correlation between normal SNe Ia LC decline rates and global ages (morphologies, colours, and masses) of their host galaxies (Tables 9 and 12). On average, those normal SNe Ia that are in galaxies above the Green Valley, i.e. in early-type, red, massive, and old hosts, have faster declining LCs in comparison with those in galaxies below the Green Valley, i.e. in late-type, blue, less massive, and younger hosts (Tables 7 and 10). The results suggest that the observed correlations between normal SNe Ia LC decline rates and global properties of host galaxies are due to a superposition of at least two distinct populations of faster and slower declining normal SNe Ia from old (halo/bulge and old disc) and young (starforming disc) components of hosts, respectively dominating in the Red Sequence and Blue Cloud of the colour-mass diagram.

(v) For the first time we show that the LC decline rates of subluminous/91bg-like $\mathrm{SNe}$ and overluminous/91T-like events do not show dependencies on the host galaxy morphology and colour (Figs. 4, 7, and Table 9). The distribution of hosts on the colourmass diagram (Fig. 6) confirms the known tendency for 91bg-like $\mathrm{SNe}$ to occur in globally red/old galaxies (from halo/bulge and old disc components) while 91T-like events prefer blue/younger hosts (related to star-forming component). Probably, the decline rates of 02cx-like SNe also do not show dependencies on hosts' properties. On average, the youngest global ages of $02 \mathrm{cx}$-like $\mathrm{SNe}$ hosts and their positions in the colour-mass diagram hint that these events likely originate from the young stellar component, but they differ from 91T-like events in the LC decline rate.

(vi) As in our previous study (Barkhudaryan et al. 2019), for the progenitors of $91 \mathrm{bg}$-like events we favour the DD channel, in particular the progenitor models such as He-ignited violent mergers
(CO WD primary with He WD companion, e.g. Pakmor et al. 2013; Crocker et al. 2017). In agreement with our findings, these models predict very long delay times for subluminous 91bg-like SNe ( $~$ several Gyr). In addition, the DD channel with CO WD primary and CO (or hybrid He-CO) WD companion (e.g. Pakmor et al. 2013; Perets et al. 2019) can provide an appropriate DTD for normal SNe Ia with an initial peak below/close to $1 \mathrm{Gyr}$ and a tail up to about 10 Gyr (e.g. Childress et al. 2014; Maoz et al. 2014). On the other hand, the DTD of the SD channel has a sharp cutoff at 2-3 Gyr (e.g. Han \& Podsiadlowski 2004) and can be appropriate for 91T-like SNe. Note that massive CO WD primaries in DD models are naturally explode in more luminous $\mathrm{SNe}$ Ia and also can be appropriate for these overluminous events (e.g. Ruiter et al. 2013). Also, we show evidence of short DTD for 02cx-like SNe, which can be interpreted within the leading model for the events (a binary system where $\mathrm{SN}$ arises from massive CO WD that quickly accretes helium from an He-star donor, and then explosion of WD does not necessarily fully disrupt the star, e.g. Postnov \& Yungelson 2014).

We stress again that, in an ideal case, the stellar population properties obtained from the SN location in the host galaxy would be preferable to the global host properties that we estimated in our study (e.g. Rigault et al. 2013; Rose et al. 2019; Panther et al. 2019). Therefore, additional local age-estimations are needed for normal and peculiar (91T-, 91bg-, and 02cx-like) SN Ia subclasses to more precisely test the DTDs of the SD and DD progenitor models. Hopefully, the forthcoming Vera C. Rubin Observatory (formerly the Large Synoptic Survey Telescope) will provide much bigger spectroscopic and photometric sample of relatively nearby SNe Ia that will give an opportunity to better constrain the nature of their progenitors.

\section{ACKNOWLEDGEMENTS}

We are grateful to our referee for his/her constructive comments. This work was supported by the RA MES State Committee of Science, in the frame of the research project number 15T-1C129. This work was made possible in part by a research grant from the Armenian National Science and Education Fund (ANSEF) based in New York, USA. MT is partially supported by the PRIN-INAF 2016 with the project Towards the SKA and CTA era: discovery, localisation, and physics of transient sources (P.I. M. Giroletti). Funding for the Sloan Digital Sky Survey IV has been provided by the Alfred P. Sloan Foundation, the US Department of Energy Office of Science, and the Participating Institutions. SDSS-IV acknowledges support and resources from the Center for High-Performance Computing at the University of Utah. The SDSS web site is www.sdss.org. SDSS-IV is managed by the Astrophysical Research Consortium for the Participating Institutions of the SDSS Collaboration including the Brazilian Participation Group, the Carnegie Institution for Science, Carnegie Mellon University, the Chilean Participation Group, the French Participation Group, Harvard-Smithsonian Center for Astrophysics, Instituto de Astrofísica de Canarias, The Johns Hopkins University, Kavli Institute for the Physics and Mathematics of the Universe (IPMU) / University of Tokyo, the Korean Participation Group, Lawrence Berkeley National Laboratory, Leibniz Institut für Astrophysik Potsdam (AIP), Max-Planck-Institut für Astronomie (MPIA Heidelberg), Max-Planck-Institut für Astrophysik (MPA Garching), Max-Planck-Institut für Extraterrestrische Physik (MPE), National Astronomical Observatories of China, New Mexico State University, New York University, University of Notre Dame, 
Observatário Nacional / MCTI, The Ohio State University, Pennsylvania State University, Shanghai Astronomical Observatory, United Kingdom Participation Group, Universidad Nacional Autónoma de México, University of Arizona, University of Colorado Boulder, University of Oxford, University of Portsmouth, University of Utah, University of Virginia, University of Washington, University of Wisconsin, Vanderbilt University, and Yale University.

\section{DATA AVAILABILITY}

The database underlying this study is available in the online version (Supporting Information) of the article. The first 10 entries of our database of 407 individual $\mathrm{SNe}$ Ia (SN designation, spectroscopic subclass, $\Delta m_{15}$, and corresponding sources of the data) and their 394 hosts (galaxy designation, distance, corrected ugriz apparent magnitudes, morphological type, and luminosity-weighted age) are shown in Table 13. The full table is available electronically in an CSV file format. A portion is shown in Table 13 for guidance regarding its content and format.

\section{REFERENCES}

Ahumada R., et al., 2020, ApJS, 249, 3

Altavilla G., et al., 2004, MNRAS, 349, 1344

Arnett W. D., 1982, ApJ, 253, 785

Ashall C., Mazzali P., Sasdelli M., Prentice S. J., 2016, MNRAS, 460, 3529

Baldry I. K., Balogh M. L., Bower R. G., Glazebrook K., Nichol R. C., Bamford S. P., Budavari T., 2006, MNRAS, 373, 469

Barkhudaryan L. V., Hakobyan A. A., Karapetyan A. G., Mamon G. A., Kunth D., Adibekyan V., Turatto M., 2019, MNRAS, 490, 718

Blondin S., et al., 2012, AJ, 143, 126

Bottinelli L., Gouguenheim L., Paturel G., Teerikorpi P., 1995, A\&A, 296, 64

Branch D., Fisher A., Nugent P., 1993, AJ, 106, 2383

Brout D., Scolnic D., 2020, preprint, (arXiv:2004.10206)

Burns C. R., et al., 2018, ApJ, 869, 56

Cameron E., 2011, PASA, 28, 128

Campbell H., Fraser M., Gilmore G., 2016, MNRAS, 457, 3470

Chambers K. C., et al., 2016, preprint, (arXiv:1612.05560)

Childress M. J., Wolf C., Zahid H. J., 2014, MNRAS, 445, 1898

Chilingarian I. V., Melchior A.-L., Zolotukhin I. Y., 2010, MNRAS, 405, 1409

Claeys J. S. W., Pols O. R., Izzard R. G., Vink J., Verbunt F. W. M., 2014, A\&A, 563, A83

Coelho R. C. V., Calvão M. O., Reis R. R. R., Siffert B. B., 2015, Eur. J. Phys., 36, 015007

Crocker R. M., et al., 2017, Nat. Astron., 1, 0135

de Lapparent V., Baillard A., Bertin E., 2011, A\&A, 532, A75

Feigelson E. D., Babu G. J., 2012, Modern Statistical Methods for Astronomy. Cambridge Univ. Press, Cambridge, UK

Filippenko A. V., et al., 1992a, AJ, 104, 1543

Filippenko A. V., et al., 1992b, ApJ, 384, L15

Finkbeiner D. P., et al., 2016, ApJ, 822, 66

Fioc M., Rocca-Volmerange B., 1997, A\&A, 326, 950

Fioc M., Rocca-Volmerange B., 1999, preprint, (arXiv: astro-ph/9912179)

Fisher R., Jumper K., 2015, ApJ, 805, 150

Folatelli G., et al., 2013, ApJ, 773, 53

Foley R. J., et al., 2009, AJ, 138, 376

Foley R. J., et al., 2013, ApJ, 767, 57

Gallagher J. S., Garnavich P. M., Berlind P., Challis P., Jha S., Kirshner R. P., 2005, ApJ, 634, 210

Gallagher J. S., Garnavich P. M., Caldwell N., Kirshner R. P., Jha S. W., Li W., Ganeshalingam M., Filippenko A. V., 2008, ApJ, 685, 752

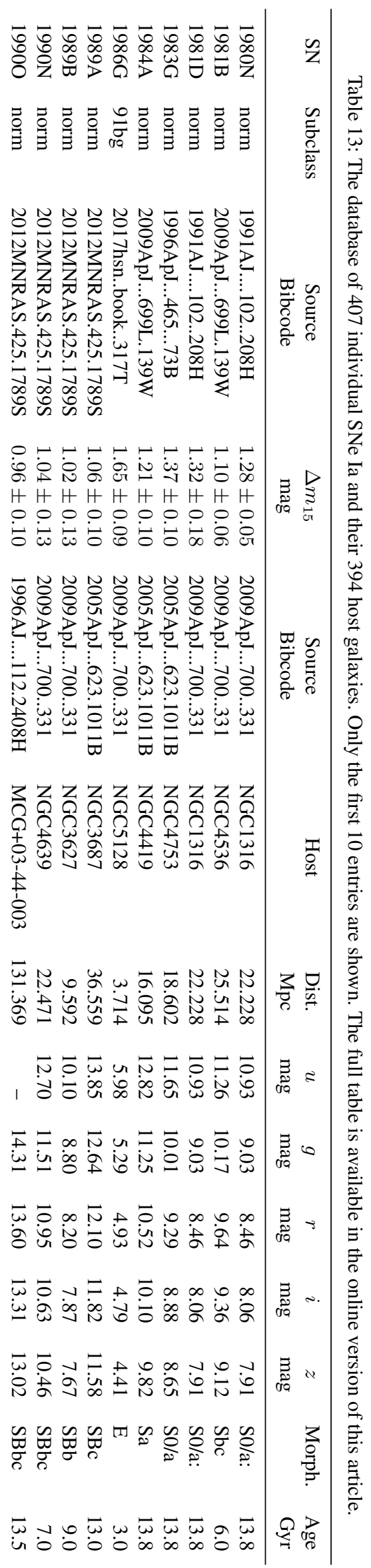


Ganeshalingam M., et al., 2010, ApJS, 190, 418

Ge X., Gu Q.-S., García-Benito R., Xiao M.-Y., Li Z.-N., 2020, ApJ, 889, 132

Gilfanov M., Bogdán Á., 2010, Nature, 463, 924

Gomes J. M., et al., 2016, A\&A, 588, A68

González Delgado R. M., et al., 2015, A\&A, 581, A103

González-Gaitán S., et al., 2011, ApJ, 727, 107

González-Gaitán S., et al., 2014, ApJ, 795, 142

Graham J., Foley R. J., 2004, IAU Circ., 8340, 1

Graur O., Bianco F. B., Modjaz M., Shivvers I., Filippenko A. V., Li W., Smith N., 2017, ApJ, 837, 121

Greggio L., 2005, A\&A, 441, 1055

Guillochon J., Parrent J., Kelley L. Z., Margutti R., 2017, ApJ, 835, 64

Gupta R. R., et al., 2011, ApJ, 740, 92

Guy J., et al., 2007, A\&A, 466, 11

Hakobyan A. A., Petrosian A. R., McLean B., Kunth D., Allen R. J., Turatto M., Barbon R., 2008, A\&A, 488, 523

Hakobyan A. A., Adibekyan V. Z., Aramyan L. S., Petrosian A. R., Gomes J. M., Mamon G. A., Kunth D., Turatto M., 2012, A\&A, 544, A81

Hakobyan A. A., et al., 2016, MNRAS, 456, 2848

Hakobyan A. A., et al., 2017, MNRAS, 471, 1390

Hamuy M., Phillips M. M., Suntzeff N. B., Schommer R. A., Maza J., Aviles R., 1996, AJ, 112, 2391

Hamuy M., Trager S. C., Pinto P. A., Phillips M. M., Schommer R. A., Ivanov V., Suntzeff N. B., 2000, AJ, 120, 1479

Han Z., Podsiadlowski P., 2004, MNRAS, 350, 1301

Hicken M., et al., 2009, ApJ, 700, 331

Hillebrandt W., Kromer M., Röpke F. K., Ruiter A. J., 2013, Front. Phys., 8, 116

Howell D. A., 2001, ApJ, 554, L193

Howell D. A., et al., 2009, ApJ, 691, 661

Iben I. J., Tutukov A. V., 1984, ApJS, 54, 335

Iben I. J., Tutukov A. V., 1985, ApJS, 58, 661

Jha S. W., 2017, in Alsabti A. W., Murdin P., eds, Type Iax Supernovae, Handbook of Supernovae. Springer, Cham, p. 375

Jha S., Riess A. G., Kirshner R. P., 2007, ApJ, 659, 122

Johansson J., et al., 2013, MNRAS, 435, 1680

Kang Y., Lee Y.-W., Kim Y.-L., Chung C., Ree C. H., 2020, ApJ, 889, 8

Karapetyan A. G., Hakobyan A. A., Barkhudaryan L. V., Mamon G. A., Kunth D., Adibekyan V., Turatto M., 2018, MNRAS, 481, 566

Kaviraj S., Peirani S., Khochfar S., Silk J., Kay S., 2009, MNRAS, 394, 1713

Kelsey L., et al., 2020, preprint, (arXiv:2008.12101)

Könyves-Tóth R., et al., 2020, ApJ, 892, 121

Lee M. G., Jang I. S., Kang J., 2019, ApJ, 871, 33

Leibundgut B., et al., 1993, AJ, 105, 301

Li W., et al., 2003, PASP, 115, 453

Li W., et al., 2011, MNRAS, 412, 1441

Lipunov V. M., Panchenko I. E., Pruzhinskaya M. V., 2011, New Astron., 16, 250

Livio M., Mazzali P., 2018, Phys. Rep., 736, 1

Maeda K., Terada Y., 2016, Int. J. Mod. Phys. D, 25, 1630024

Maoz D., Mannucci F., Nelemans G., 2014, ARA\&A, 52, 107

Matteucci F., Greggio L., 1986, A\&A, 154, 279

Mazzali P. A., Röpke F. K., Benetti S., Hillebrandt W., 2007, Science, 315, 825

McIntosh D. H., et al., 2014, MNRAS, 442, 533

Mendez A. J., Coil A. L., Lotz J., Salim S., Moustakas J., Simard L., 2011, ApJ, 736, 110

Meng X.-C., 2019, ApJ, 886, 58

Neill J. D., et al., 2009, ApJ, 707, 1449

Nomoto K., Iwamoto K., Kishimoto N., 1997, Science, 276, 1378

Onken C. A., et al., 2019, PASA, 36, e033

Oohama N., Okamura S., Fukugita M., Yasuda N., Nakamura O., 2009, ApJ, 705,245

Pakmor R., Kromer M., Taubenberger S., Springel V., 2013, ApJ, 770, L8

Pan Y. C., et al., 2014, MNRAS, 438, 1391

Panther F. H., Seitenzahl I. R., Ruiter A. J., Crocker R. M., Lidman C., Wang E. X., Tucker B. E., Groves B., 2019, PASA, 36, e031
Perets H. B., et al., 2010, Nature, 465, 322

Perets H. B., Zenati Y., Toonen S., Bobrick A., 2019, preprint, (arXiv:1910.07532)

Perley D. A., et al., 2020, preprint, (arXiv:2009.01242)

Perlmutter S., et al., 1999, ApJ, 517, 565

Phillips M. M., 1993, ApJ, 413, L105

Phillips M. M., Wells L. A., Suntzeff N. B., Hamuy M., Leibundgut B., Kirshner R. P., Foltz C. B., 1992, AJ, 103, 1632

Phillips M. M., Lira P., Suntzeff N. B., Schommer R. A., Hamuy M., Maza J., 1999, AJ, 118, 1766

Ponder K. A., Wood-Vasey W. M., Weyant A., Barton N. T., Galbany L., Garnavich P., Matheson T., 2020, preprint, (arXiv:2006.13803)

Postnov K. A., Yungelson L. R., 2014, Living Rev. Relativ., 17, 3

Pruzhinskaya M., Novinskaya A., Pauna N., Rosnet P., 2020, preprint, (arXiv:2006.09433)

Riess A. G., et al., 1998, AJ, 116, 1009

Rigault M., et al., 2013, A\&A, 560, A66

Roman M., et al., 2018, A\&A, 615, A68

Rose B. M., Garnavich P. M., Berg M. A., 2019, ApJ, 874, 32

Ruiter A. J., 2020, preprint, (arXiv:2001.02947)

Ruiter A. J., Belczynski K., Fryer C., 2009, ApJ, 699, 2026

Ruiter A. J., et al., 2013, MNRAS, 429, 1425

Schawinski K., 2009, MNRAS, 397, 717

Schawinski K., et al., 2014, MNRAS, 440, 889

Schlafly E. F., Finkbeiner D. P., 2011, ApJ, 737, 103

Schlegel D. J., Finkbeiner D. P., Davis M., 1998, ApJ, 500, 525

Shen K. J., Toonen S., Graur O., 2017, ApJ, 851, L50

Siebert M. R., et al., 2019, MNRAS, 486, 5785

Silverman J. M., et al., 2012, MNRAS, 425, 1789

Stahl B. E., et al., 2019, MNRAS, 490, 3882

Stritzinger M. D., et al., 2015, A\&A, 573, A2

Suh H., Yoon S.-c., Jeong H., Yi S. K., 2011, ApJ, 730, 110

Sullivan M., et al., 2010, MNRAS, 406, 782

Taubenberger S., 2017, in Alsabti A. W., Murdin P., eds, The Extremes of Thermonuclear Supernovae, Handbook of Supernovae. Springer, Cham, p. 317

Taubenberger S., et al., 2011, MNRAS, 412, 2735

Taylor E. N., et al., 2011, MNRAS, 418, 1587

Turatto M., Benetti S., Cappellaro E., Danziger I. J., Della Valle M., Gouiffes C., Mazzali P. A., Patat F., 1996, MNRAS, 283, 1

Uddin S. A., Mould J., Lidman C., Ruhlmann-Kleider V., Zhang B. R., 2017, ApJ, 848, 56

Uddin S. A., et al., 2020, preprint, (arXiv:2006.15164)

Valenti S., et al., 2009, Nature, 459, 674

Whelan J., Iben I. J., 1973, ApJ, 186, 1007

White C. J., et al., 2015, ApJ, 799, 52

Wild P., 1994, IAU Circ., 5982, 2

Wolf C., et al., 2018, PASA, 35, e010

Yaron O., Gal-Yam A., 2012, PASP, 124, 668

Yungelson L. R., Livio M., 2000, ApJ, 528, 108

Zapartas E., et al., 2017, A\&A, 601, A29

Zenati Y., Toonen S., Perets H. B., 2019, MNRAS, 482, 1135

\section{SUPPORTING INFORMATION}

Supplementary data are available at MNRAS online.

\section{PaperVIIonlinedata.csv}

Please note: Oxford University Press is not responsible for the content or functionality of any supporting materials supplied by the authors. Any queries (other than missing material) should be directed to the corresponding author for the article.

This paper has been typeset from a $\mathrm{T}_{\mathrm{E}} \mathrm{X} / \mathrm{L}_{\mathrm{A}} \mathrm{T}_{\mathrm{E}} \mathrm{X}$ file prepared by the author. 